\title{
Applying the MIDAS Touch: How to Handle Missing Values in Large and Complex Data*
}

\author{
Ranjit Lall ${ }^{\dagger} \quad$ Thomas Robinson ${ }^{\ddagger}$
}

April 2, 2020

\begin{abstract}
Principled methods for analyzing missing values, based chiefly on multiple imputation, have become increasingly popular among political scientists yet can struggle to handle the kinds of large and complex data that are also becoming common. We propose an accurate, fast, and scalable approach to multiple imputation, which we call MIDAS (Multiple Imputation with Denoising Autoencoders). MIDAS employs a class of unsupervised neural networks known as denoising autoencoders, which are designed to reduce dimensionality by corrupting and attempting to reconstruct a subset of data. We repurpose denoising autoencoders for multiple imputation by treating missing values as an additional portion of corrupted data and drawing imputations from a model trained to minimize the reconstruction error on the originally observed portion. Systematic tests involving simulated as well as real political data illustrate MIDAS's accuracy and efficiency across a range of settings. We provide open-source software for implementing MIDAS.
\end{abstract}

${ }^{*}$ For valuable feedback on previous drafts, we are grateful to Matthew Blackwell, Andrew Eggers, Jeff Gill, Kosuke Imai, Gary King, Walter Mattli, and Alex Stenlake. We also thank Kin Wai (Keith) Chan, James Honaker, Ankur Moitra, and Devrarat Shah for helpful conversations on technical aspects of this research and Jonathan Kropko for generously sharing replication code. Earlier versions of the paper were presented at the Quantitative Social Science Colloquium at Princeton University and the Artificial Intelligence and Machine Learning Society at the University of Queensland. The methods described in the paper are implemented through the open-source software midas, which is available as a class in the Python programming environment (https://github.com/ranjitlall/MIDAS). midas was developed by Ranjit Lall, Thomas Robinson, and Alex Stenlake.

${ }^{\dagger}$ London School of Economics and Political Science. Email: r.lall@lse.ac.uk.

${ }^{\ddagger}$ University of Oxford. Email: thomas.robinson@politics.ox.ac.uk. 


\section{Introduction}

In recent years, the analysis of political science data with missing values has been characterized by two trends that have yet to be reconciled. First, to avoid the problems caused by popular ad-hoc methods such as listwise deletion (discarding rows of the dataset that contain any missing values), scholars are increasingly turning to principled techniques for imputing, or filling in, missing values recommended by the statistics community. The most widely used of these techniques, multiple imputation (MI), involves replacing each missing element with several values that preserve relationships within the observed data while representing uncertainty about the correct value. In the words of a prominent scholar of missing-data analysis, "[MI] is now accepted as the best general method to deal with incomplete data in many fields" (van Buuren 2012, 25).

Second, advances in computational power, efficiency, and storage capacity have enabled political scientists to compile and analyze unprecedentedly large and complex datasets, ushering in an era of so-called "Big Data" in the discipline. While massively increasing the amount of information available for analysis, however, this development has not eliminated the problem of missing data. That is, bigger data has not necessarily translated into more complete data.

The growing scale and complexity of political science data present a computational challenge for existing MI algorithms, which were generally designed for small or mediumsized applications with relatively simple (mostly linear) structures. While working well in many settings, these algorithms can suffer from performance problems when applied to larger datasets with features such as high dimensionality, severe nonlinearities, and unconventional functional forms. Convergence failures and slow - sometimes prohibitive runtimes become increasingly common, with imputations more likely to take on extreme and unusual values. Analysts of such datasets can thus face an unappealing choice: restrict 
the imputation model to a subset of rows or columns, risking bias and reducing statistical efficiency; or employ an ad-hoc method that can be applied to the entire dataset, such as listwise deletion or mean imputation (replacing missing data with observed column averages), creating an even greater risk of bias and guaranteeing inefficiency.

We propose an approach that enables political scientists to accurately and efficiently multiply impute missing values in large and complex datasets, which we call MIDAS (Multiple Imputation with Denoising Autoencoders). MIDAS employs a class of unsupervised neural networks known as denoising autoencoders (DAs), which were recently developed to optimize the task of dimensionality reduction. DAs corrupt a subset of input data via the injection of stochastic noise and attempt to reconstruct it through a series of nested nonlinear transformations. The key innovation in MIDAS is to treat missing values as an additional portion of corrupted data and thus draw imputations from a model trained to minimize the reconstruction error on the originally observed portion. To reduce the risk of overfitting, we train this imputation-repurposed DA with the technique of dropout, which extends the corruption process deeper into the neural network architecture. With the combination of denoising and dropout, MIDAS employs an effectively nonparametric imputation model that places constraints not on the joint distribution of the data - the standard approach to MI - but only on the distribution of possible functions that characterize the data. This enables the model to capture highly complex relationships between variables, giving MIDAS the flexibility to approximate the performance of existing MI strategies with simple data structures while delivering gains in more complex settings.

To implement MIDAS, we develop a fast and scalable algorithm that markedly expands the range and quantity of data that can be analyzed with MI. This procedure leverages the efficient and adaptable computational architecture of the TensorFlow programming platform, allowing a wide variety of data types and supporting high degrees of parallelization on supported systems. As a companion to this study, we make the algorithm avail- 
able in an easy-to-use class in the Python programming environment (midas) - the first full-featured, open-source software for performing MI with neural network technology:1 Further information on the software is provided in Appendices 1 and 2 .

We illustrate MIDAS's accuracy and scalability through a series of systematic tests involving real as well as simulated data. We first conduct two Monte Carlo simulation experiments that assess MIDAS's accuracy under the statistical conditions assumed by the dominant approach to MI, namely, joint multivariate normality. The first experiment establishes that MIDAS yields accurate estimated posterior densities and confidence intervals for linear regression coefficients, while the second shows that the accuracy of MIDAS's imputed values and parameter estimates compares favorably with that of leading existing MI algorithms. We then move to a more realistic setting, introducing varying levels and patterns of missingness into a census dataset frequently used for testing machine learning algorithms. We find that MIDAS yields more accurate imputed values than other MI algorithms across most missingness conditions, even performing well under patterns where MI cannot avoid some degree of bias.

We test MIDAS's scalability by sampling increasing numbers of rows and columns from a popular electoral survey that typifies the kind of large and complex data analyzed by political scientists. MIDAS produces completed datasets in consistently less time than existing MI algorithms, with the gap increasing linearly with the number of rows and exponentially with the number of columns. Even with modestly-sized datasets, MIDAS's efficiency translates into substantial time savings for analysts. For datasets approaching the dimensions of modern Big Data, where the time taken by existing MI algorithms can render analysis infeasible, it may make the difference between employing a principled, statistically valid approach to analyzing missing data and resorting to an ad-hoc method that could result in

\footnotetext{
${ }^{1}$ The software is accompanied by a comprehensive online guide to its functions and arguments, interactive Jupyter Notebook examples of how to apply MIDAS to real data, and a set of diagnostic tools to help users calibrate the technique for their specific application.
} 
biased and inefficient inferences.

Finally, we provide an applied illustration of MIDAS's capacity to handle datasets that pose computational problems for existing MI algorithms - that is, to give us access to new substantive knowledge - that involves estimating the latent ideology of participants in the electoral survey used in the scalability test. We show that substituting MIDAS for listwise deletion, which enables us to recover estimates for more than 10,000 additional respondents, materially alters our understanding of the distribution of latent ideology in the sample and of the relationship between this variable and presidential job approval.

\section{MIDAS: Theory and Implementation}

\section{Multiple Imputation}

The first building block of MIDAS, MI, consists of three steps: (1) replacing each missing element in the dataset with $M$ independently drawn imputed values that preserve relationships expressed by observed elements; (2) analyzing the $M$ completed datasets separately and estimating parameters of interest; and (3) combining the $M$ separate parameter estimates using a simple set of rules that leverages variation across these datasets to reflect our uncertainty about the correct imputation model.2]

The dominant approach to MI assumes that the complete data follow a multivariate normal distribution, which implies that each variable is continuous and a linear function of all others (e.g., King et al. 2001; Honaker and King 2010). An alternative approach models each variable's distribution conditionally on all others in an iterative fashion, typically using a generalized linear estimator, which allows for a wider class of variable types and distributions (e.g., Kropko et al. 2014). Imputed values, however, need not be drawn

\footnotetext{
${ }^{2}$ These rules, which are described in Rubin (1987), involve averaging the $M$ parameter estimates and computing variance as a weighted sum of the estimated variance within and between the $M$ datasets.
} 
from a posterior density. A notable nonparametric approach is predictive mean matching, which involves replacing missing values with observed ones from similar rows (according to a chosen metric) (e.g., Cranmer and Gill 2013).

All approaches to MI share three attractive features. First, they yield unbiased parameter estimates under a fairly wide range of statistical conditions: data are either missing completely at random (MCAR), i.e., the pattern of missingness is independent of observed and missing data, or missing at random (MAR), i.e., this pattern depends on observed data. They cannot avoid bias when data are missing not at random (MNAR), i.e., missingness depends on missing data, though can still perform well if the observed data include strong predictors of missingness (Lall 2016; Mustillo and Kwon 2015) 3] Second, they are statistically efficient because they utilize all observed values in the dataset (whether or not they are part of the subsequent analytical model). Third, from a practical perspective, they are simple to implement because they do not require directly modeling the missingness mechanism and, due to the separation between imputation and analysis, can be combined with standard complete-data methods.

Although useful in many settings, existing approaches to MI also have a common limitation: they can perform poorly with the kinds of large and complex datasets that are becoming increasingly common in political science. This is in part because extreme departures from their assumptions are more common in these datasets and in part due to problems of computational implementation. Most approaches are implemented with a variant of either the imputation-posterior algorithm, which draws missing values from the appropriate posterior distribution using Markov chain Monte Carlo (MCMC) methods, or the expectation-maximization algorithm, a similar procedure that substitutes maximum likelihood estimates for posterior draws. For a variety of reasons - including their serial nature, sweep of the entire dataset at each iteration, and simultaneous updating of all

\footnotetext{
${ }^{3}$ For formal definitions of these missingness mechanisms, see Little and Rubin (2002).
} 
parameters — both algorithms "have well-known problems with large data sets...creating unacceptably long run-times or software crashes" (Honaker and King $\mid 2010,564)$. Even when they do converge, they can fail to accurately approximate posteriors due to local maxima or major divergence from the assumed joint distribution. Some approaches seek to overcome these problems by combining one of the above algorithms with bootstrapping (i.e., random sampling with replacement). As each bootstrapped sample is the same size as the original dataset, however, these routines can also slow down sharply or fail to converge when applied to large datasets. We later provide systematic evidence of these scalability issues.

\section{Denoising Autoencoder Neural Networks}

MIDAS implements MI with the aid of artificial neural networks, a concept inspired by the structure of the human brain that has been used to enhance the accuracy and efficiency of a wide array of computational tasks.4 A neural network consists of a series of nested nonlinear functions usually depicted as interconnected nodes organized in layers. Input data are fed into the network through an input layer, processed by nodes in one or more hidden layers, and returned via nodes in an output layer. To more precisely describe these models, we adopt the linear algebraic notation typically used in the machine learning literature, which allows for concise expression of deeply nested functions: italicized upper-case symbols denote random vectors (e.g., $X$ ); bold lower-case symbols (x) denote ordinary column vectors, i.e., realizations of random vectors; bold upper-case symbols denote matrices, with $\mathbf{D}=\left\{\mathbf{D}_{o b s}, \mathbf{D}_{\text {mis }}\right\}$ denoting a dataset in which $\mathbf{D}_{o b s}$ is observed and $\mathbf{D}_{m i s}$ is missing; and superscripts in parentheses index hidden layers of a network.

The model for a "forward pass" - or computation of output values given input data -

${ }^{4}$ For an overview of the evolution of neural networks and deep learning, see Goodfellow et al. (2016, Chapter $1)$. 
through layer $h$ of a neural network is:

$$
\mathbf{y}^{(h)}=\sigma\left(\mathbf{W}^{(h)} \mathbf{y}^{(h-1)}+\mathbf{b}^{(h)}\right)
$$

where $\mathbf{y}^{(h)}$ is a vector of outputs from layer $h\left(\mathbf{y}^{(0)}=\mathbf{x}\right.$ is the input), $\mathbf{W}^{(h)}$ is a matrix of weights connecting the nodes in layer $h-1$ with the nodes in layer $h, \mathbf{b}$ is a vector of biases for layer $h$, and $\sigma$ is a nonlinear activation function. The introduction of nonlinearity into the model enables neural networks to efficiently learn highly complex functional forms with few hidden layers. This model can be generalized to an arbitrary number of hidden layers $H$ :

$$
\mathbf{y}=\Phi\left(\mathbf{W}^{(H)}\left[\ldots\left[\sigma\left(\mathbf{W}^{(2)}\left[\sigma\left(\mathbf{W}^{(1)} \mathbf{x}+\mathbf{b}^{(1)}\right)\right]+\mathbf{b}^{(2)}\right)\right] \ldots\right]+\mathbf{b}^{(H)}\right)
$$

where $\mathbf{x}$ is a vector of inputs and $\Phi$ is a final-layer activation function that returns outputs with the appropriate distribution.

The parameters of the network $(\theta)$ are weights and biases, which are trained to minimize a loss function $L(\mathbf{y}, \hat{\mathbf{y}})$ that measures the distance between actual and predicted outputs. Training involves four steps, collectively known as an epoch, which are repeated until some convergence criterion is met: (1) performing a forward pass through the network using current $\theta$; (2) calculating $L$; (3) using the chain rule to calculate error gradients with respect to weights in each layer, a technique called backpropagation (Rumelhart et al. $1986 \mathrm{~b}$ a); and (4) adjusting weights in the direction of the negative gradient for the next forward pass. Characteristics such as the number of training cycles per epoch, which are specified by the analyst rather than learned in training, are referred to as hyperparameters.

One class of neural networks that is naturally suited to the task of imputing missing data is the DA, an extension of the classical autoencoder - a well-established tool for dimensionality reduction in machine learning — proposed by Vincent et al. (2008). Classical 
autoencoders consist of two parts. First, an encoder deterministically maps an input vector $\mathrm{x}$ to a lower-dimensional representation $\mathrm{y}$ by compressing it through a series of shrinking hidden layers that culminate in a "bottleneck" layer (indexed by $B$ ):

$$
\mathbf{y}=f_{\theta}(\mathbf{x})=\sigma\left(\mathbf{W}^{(B)}\left[\ldots\left[\sigma\left(\mathbf{W}^{(2)}\left[\sigma\left(\mathbf{W}^{(1)} \mathbf{x}+\mathbf{b}^{(1)}\right)\right]+\mathbf{b}^{(2)}\right)\right] \ldots\right]+\mathbf{b}^{(B)}\right)
$$

Second, a decoder maps $\mathbf{y}$ back to a reconstructed vector $\mathbf{z}$ with the same probability distribution and dimensions as $\mathrm{x}$ by passing it through a parallel series of expanding hidden layers culminating in the output layer:

$$
\mathbf{z}=g_{\theta^{\prime}}(\mathbf{y})=\Phi\left(\mathbf{W}^{(H) \prime}\left[\ldots\left[\sigma\left(\mathbf{W}^{(B+2) \prime}\left[\sigma\left(\mathbf{W}^{(B+1) \prime} \mathbf{y}+\mathbf{b}^{(B+1) \prime}\right)\right]+\mathbf{b}^{(B+2) \prime}\right)\right] \ldots\right]+\mathbf{b}^{(H) \prime}\right)
$$

To map $\mathrm{z}$ as closely as possible to $\mathrm{x}$, weights are adjusted by backpropagation to minimize a loss function $L(\mathbf{x}, \mathbf{z})$. This process yields a latent representation that captures the key axes of variation in $\mathrm{x}$ in a similar manner to principal component analysis.

DAs were developed to prevent autoencoders from learning an identical representation of the input (the identity function) while enabling them to extract more robust features from the data, that is, features that generalize better to new samples from the same datagenerating process. They achieve these benefits by partially corrupting inputs through the injection of stochastic noise: $\mathbf{x} \rightarrow \tilde{\mathbf{x}} \sim q_{D}(\mathbf{x} \mid \tilde{\mathbf{x}})$. The corrupted input is then mapped to a hidden representation $\mathbf{y}=f_{\theta}(\tilde{\mathbf{x}})$, from which a clean or "denoised" version $\mathbf{z}=g_{\theta^{\prime}}(\mathbf{y})$ is reconstructed. Unlike before, however, $\mathrm{z}$ is now a deterministic function of $\tilde{\mathbf{x}}$ (not $\mathbf{x}$ ).

The most common corruption process involves setting a random subset of inputs to 0 . In attempting to recover these elements, the DA effectively performs a form of imputation: predicting corrupted (missing) elements based on relationships among uncorrupted (observed) elements. That is, missing values can be seen as a special case of corrupted input data. Building on this insight, recent studies have developed application-specific models 
for imputing missing values with DAs, reporting impressive performance (e.g., BeaulieuJones and Greene 2016; Duan et al.2014). These studies, however, neither offer a general model of DA-based imputation nor combine DAs with MI, forgoing the latter's advantages vis-á-vis single imputation in bias reduction, efficiency, and uncertainty representation.

To our knowledge, the only existing attempt to implement MI using DAs comes from Gondara and Wang (2018), who propose a model in which data are provisionally completed using mean imputation (for continuous variables) or mode imputation (for categorical variables) before being stochastically corrupted and passed into the DA.5 While Gondara and Wang offer only a brief overview of the model and do not make their full code publicly available, this approach appears to suffer from three limitations. First, its loss functions fail to distinguish between originally observed and originally missing values, causing reconstruction error to be measured against the mean/mode imputations, which typically result in biased parameter estimates. Second, it injects stochastic noise into inputs once rather than in each training epoch, increasing the risk of overfitting and reducing model robustness. Third, instead of sampling from a single trained network, it trains a different network for each set of imputations, substantially slowing runtime — storing all trained models and imputations in memory is computationally demanding - without improving performance. In the rest of the section, we present an alternative approach to multiply imputing missing data with DAs that avoids these issues.

\section{The MIDAS Model}

MIDAS modifies the standard DA model in two key ways. First, in addition to a random subset of inputs, it forces all missing values to 0 . The task of the DA is thus to predict corrupted values that were both originally missing $\left(\tilde{\mathbf{x}}_{\text {mis }}\right)$ and originally observed ( $\left.\tilde{\mathbf{x}}_{\text {obs }}\right)$ using a loss function that only measures the reconstruction error on the latter.

\footnotetext{
${ }^{5}$ We developed MIDAS independently and without knowledge of Gondara and Wang's research.
} 
FIGURE 1. MIDAS Neural Network Architecture

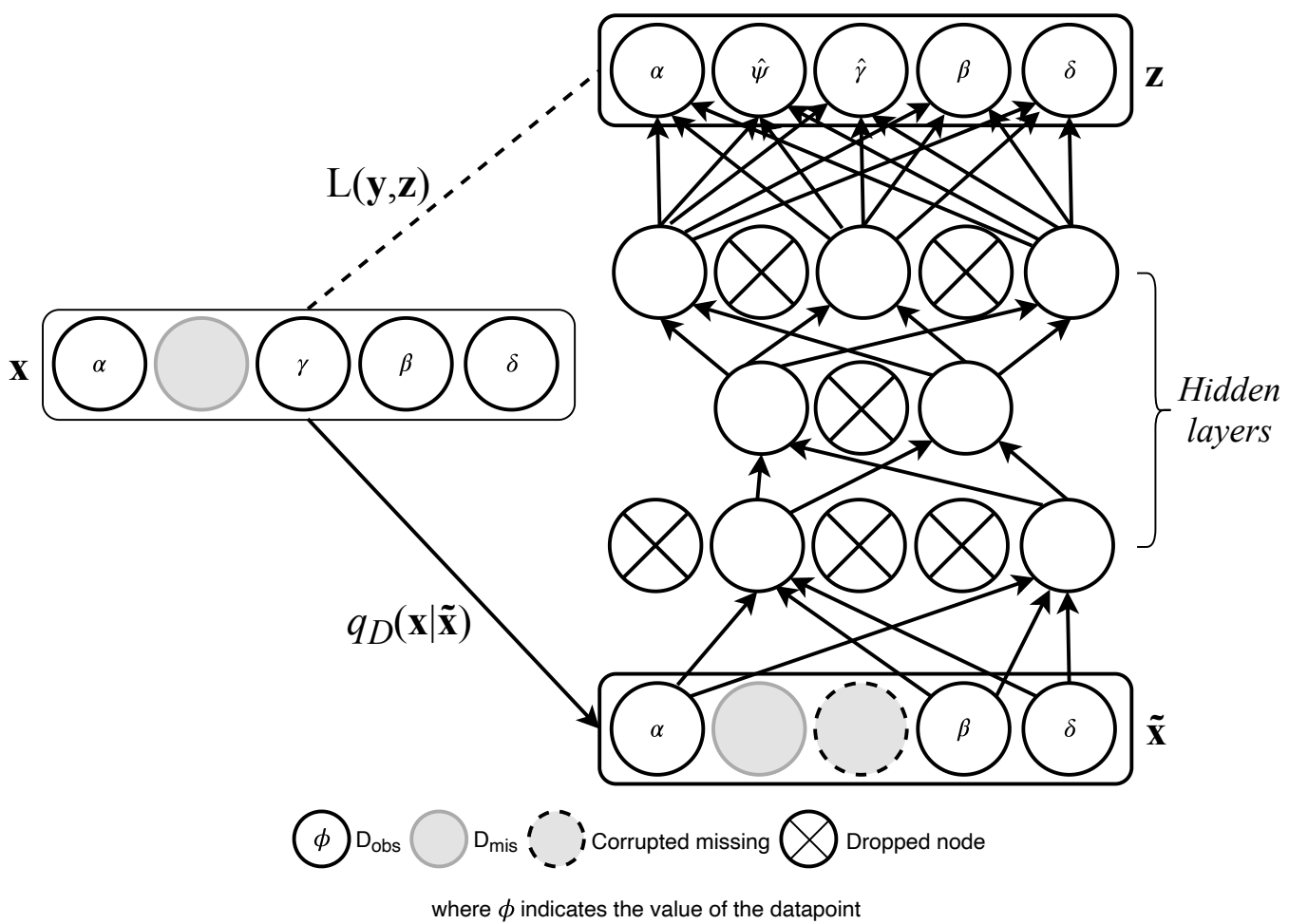

$\mathbf{x}$ is a vector of inputs, $q_{D}(\mathbf{x} \mid \tilde{\mathbf{x}})$ is the corruption process, and $\mathbf{z}$ is the "denoised" version of $\tilde{\mathbf{x}}$.

Second, to further reduce the risk of overfitting, MIDAS regularizes the DA with the complementary technique of dropout. Introduced by Hinton et al. (2012), dropout involves randomly removing (or "dropping") nodes in the hidden layers of a network during training, typically by multiplying outputs from each of these layers by a Bernoulli vector $\mathbf{v}$ that takes a value of 1 with probability $p: \tilde{\mathbf{y}}^{(h)}=\mathbf{v}^{(h)} \mathbf{y}^{(h)}, \mathbf{v}^{(h)} \sim \operatorname{Bernoulli}(p)$. Dropout is thus a generalization of the idea behind DAs, extending stochastic corruption to the hidden layers and hence enabling the extraction of even more robust features.

Dropout training proceeds by sampling an arbitrary number of "thinned" networks, with a different set of nodes dropped in each iteration. At test time, Hinton et al. propose scaling the weights of a single unthinned network by the probability that their originating nodes were retained during training. To produce multiple imputations, MIDAS instead samples $M$ thinned networks. This procedure has recently received a powerful indepen- 
dent justification from Gal and Ghahramani (2016), who show through simulation experiments that it results in more accurate parameter estimation with no additional model complexity or training time. Notably, they also prove that dropout training is mathematically equivalent to a Bayesian variational approximation of a Gaussian process (GP), a commonly used probability distribution over functions.6 The implication is that MIDAS posits not a joint distribution of the data but a distribution over possible functions that describe the data. Since GP models can estimate any continuous function arbitrarily well they are usually considered nonparametric because they have a potentially infinite number of parameters - MIDAS can thus capture a wider class of joint distributions than existing approaches to MI without making any additional parametric assumptions.

The encoder of an imputation-generating DA trained with dropout - a MIDAS network — can thus be described as:

$$
\tilde{\mathbf{y}}=f_{\theta}(\tilde{\mathbf{x}})=\sigma\left(\mathbf{W}^{(B)} \mathbf{v}^{(B)}\left[\ldots\left[\sigma\left(\mathbf{W}^{(2)} \mathbf{v}^{(2)}\left[\sigma\left(\mathbf{W}^{(1)} \tilde{\mathbf{x}}+\mathbf{b}^{(1)}\right)\right]+\mathbf{b}^{(2)}\right)\right] \ldots\right]+\mathbf{b}^{(B)}\right) .
$$

The decoder, in turn, becomes:

$$
\mathbf{z}=g_{\theta^{\prime}}(\tilde{\mathbf{y}})=\Phi\left(\mathbf{W}^{(H) \prime}\left[\ldots\left[\sigma\left(\mathbf{W}^{(B+2) \prime}\left[\sigma\left(\mathbf{W}^{(B+1) \prime} \tilde{\mathbf{y}}+\mathbf{b}^{(B+1) \prime}\right)\right]+\mathbf{b}^{(B+2) \prime}\right)\right] \ldots\right]+\mathbf{b}^{(H) \prime}\right)
$$

where $g \dot{\sim}$ GP and $\mathrm{z}$ represents a fully observed vector containing predictions of $\tilde{\mathbf{x}}_{\text {obs }}$ and $\tilde{\mathbf{x}}_{\text {mis. }}$. To produce a completed dataset, predictions of $\tilde{\mathbf{x}}_{\mathrm{mis}}$ are substituted for $\mathbf{x}_{\mathrm{mis}}$ in $\mathbf{D}$. The full architecture of a MIDAS network is illustrated in Figure 10]

The default activation function in MIDAS is exponential linear unit (ELU), which is

\footnotetext{
${ }^{6}$ Specifically, a finite vector of inputs $\mathbf{x}$ is a GP if the vector of function values $\mathbf{f}=f\left(x_{1}\right), f\left(x_{2}\right), \ldots, f\left(x_{n}\right)$ follows a multivariate normal distribution. Variational approximation is a Bayesian approach to approximating complex posteriors that involves positing a family of potential distributions and finding the member $Q$ that minimizes the Kullback-Leibler divergence to the true posterior $P$. For an introduction to this tool, see Grimmer (2010).

${ }^{7} \mathrm{~A}$ more detailed description of the MIDAS model's objective function is provided in Appendix $3 \mathrm{~A}$.
} 
known to facilitate efficient training in deep neural networks. The final-layer activation function is chosen according to the distribution of the input data $\mathrm{x}$, with identity, logistic, and softmax functions assigned to continuous, binary, and categorical variables, respectively. Loss functions take the same form as in a regular DA, measuring the distance between $\mathbf{x}$ and $\mathbf{z}: L(\mathbf{x}, \mathbf{z})$. As we are only interested in the reconstruction error for predictions of originally observed corrupted values $\left(\tilde{\mathrm{x}}_{\mathrm{obs}}\right)$, however, these functions are multiplied by a missingness indicator vector $\mathrm{r} 8$ MIDAS employs root mean squared error (RMSE) and cross-entropy loss functions for continuous and categorical variables, respectively:

$$
L(\mathbf{x}, \mathbf{z}, \mathbf{r})= \begin{cases}{\left[\frac{1}{J} \sum_{j=1}^{J} \mathbf{r}_{j}\left(\mathbf{x}_{j}-\mathbf{z}_{j}\right)^{2}\right]^{\frac{1}{2}}} & \text { if } \mathbf{x} \text { is continuous } \\ -\frac{1}{J} \sum_{j=1}^{J} \mathbf{r}_{j}\left[\mathbf{x}_{j} \log \mathbf{z}_{j}+\left(1-\mathbf{x}_{j}\right) \log \left(1-\mathbf{z}_{j}\right)\right] & \text { if } \mathbf{x} \text { is categorical. }\end{cases}
$$

In sum, unlike most existing approaches to MI, MIDAS does not assume a joint distribution of the data and use an iterative method to draw imputed values from the posterior of this distribution. Rather, it uses neural networks to "learn" the form of the data by fitting a series of nonlinear functions - in effect, a nonparametric model that only constrains the range of functions that are consistent with the data - which enables it to capture highly complex patterns. This is implemented by introducing additional missingness into the data in each training epoch, minimizing the reconstruction error for predictions of these (originally observed) corrupted values, and drawing imputations from the trained network. Under the relatively simple conditions assumed by existing approaches to MI, MIDAS should thus possess the flexibility to yield comparable accuracy to these strategies, particularly when there are strong associations between variables. As we move to more complex settings, the method should provide clear performance gains.

\footnotetext{
${ }^{8} \mathrm{~A}$ vector the same length as $\mathrm{x}$ whose elements are a 1 if the corresponding entry in $\mathbf{x}$ is observed and a 0 if it is missing.
} 
FIGURE 2. Schematic of MIDAS Training Steps

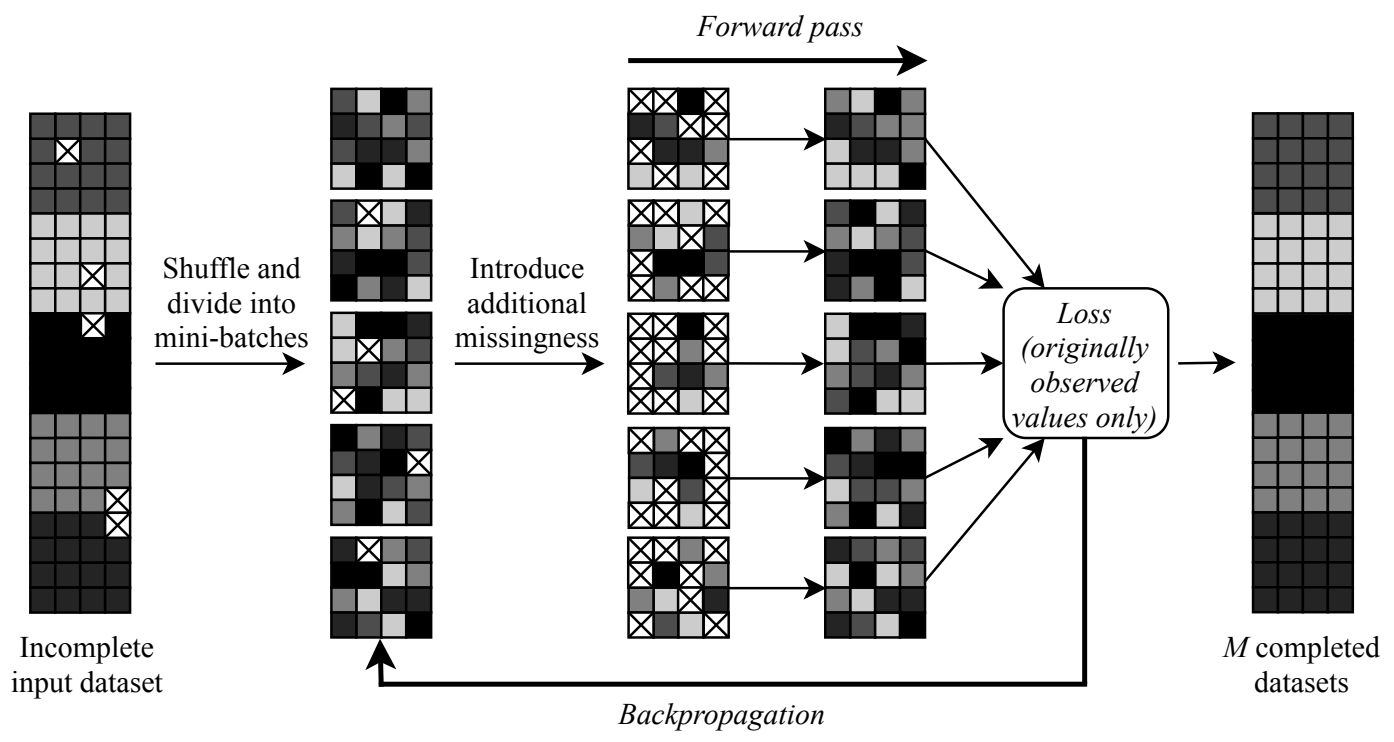

Shaded blocks represent data points (shades denote different variables); crosses indicate missing values.

\section{Algorithm}

The algorithm we have developed to implement MIDAS takes an incomplete dataset $\mathbf{D}$ as its input and returns $M$ completed datasets.9 The algorithm proceeds in three stages, each of which comprises a number of smaller steps.

In the first stage, the input data $\mathrm{D}$ are prepared for training. A missingness indicator matrix $\mathbf{R}$ is constructed for $\mathbf{D}$, allowing us to later distinguish between $\mathbf{D}_{\text {mis }}$ and $\mathbf{D}_{\text {obs }}$, and all elements of $\mathbf{D}_{\text {mis }}$ are set to 0 . A DA is then initialized according to the dimensions of D; the default architecture is a five-layer network with 256 nodes per layer. To avoid the problem of extreme gradients in deep neural network training, original weights are drawn from a truncated normal distribution using a variant of Xavier Initialization (Glorot and Bengio 2010).

\footnotetext{
${ }^{9}$ Appendix 1 provides a brief demonstration of the midas class in Python, which executes the algorithm. Appendix 2 describes two diagnostic tools that can be used to assess the performance of the imputation model (including the risk of overfitting) and calibrate hyperparameters: the technique of "overimputation" and the generation of new observations using a variational autoencoder component.
} 
In the training stage, the following five steps are repeated (see Figure 2 for a visual schematic and Appendix $3 \mathrm{~B}$ for a more formal description):

1. $\mathbf{D}$ and $\mathbf{R}$ are shuffled and sliced row-wise into paired mini-batches $\left(\mathcal{B}_{1}, \mathcal{B}_{2}, \ldots, \mathcal{B}_{n}\right)$ to accelerate convergence.

2. Mini-batch inputs are partially corrupted through multiplication by a Bernoulli vector $\mathbf{v}$ (default $p=0.8$ ).

3. In line with standard implementations of dropout, outputs from half of the nodes in hidden layers are corrupted using the same procedure.

4. A forward pass through the DA is conducted and the reconstruction error on predictions of $\tilde{\mathbf{x}}_{\mathrm{obs}}$ is calculated using the loss functions defined in Equation 7. Categorical variables must be "one-hot" encoded (i.e., converted into separate dummy variables for each unique class) before being passed into the DA.

5. Loss values are aggregated into a single term and backpropagated through the DA, with the resulting error gradients used to adjust weights for the next epoch. The size of this adjustment is controlled by a learning rate hyperparameter, $\gamma$, by which loss function derivatives are multiplied. Mini-batch stochastic gradient descent is implemented with the widely used Adam algorithm (Kingma and Ba 2015).

Finally, once training is complete, the whole of $\mathbf{D}$ is passed into the DA, which attempts to reconstruct all (i.e., originally observed and originally missing) corrupted values. A completed dataset is then constructed by replacing $D_{\text {mis }}$ with predictions of the originally missing values from the network's output. This stage is repeated $M$ times.

\section{Accuracy Tests}

How does MIDAS perform in practice? The next two sections present tests of the method's accuracy and scalability involving both simulated and real data. We begin with two tough 
simulation tests that gauge MIDAS's accuracy under the multivariate normal conditions assumed by the dominant approach to MI (without building a linearity constraint into the MIDAS model). The first is the "MAR-1" experiment originally conducted by King et al. (2001), which assesses whether MIDAS generates correct estimates of linear regression parameters; the second is the continuous component of a more general test conducted by Kropko et al. (2014), which additionally assesses the accuracy of MIDAS's imputed values. The third part of the section tests MIDAS's performance on similar metrics in a more realistic context by simulating a variety of missingness conditions in a popular census dataset.

\section{MAR-1 Experiment}

The MAR-1 experiment involves simulating 100 datasets containing 500 rows and five (moderately correlated) standardized variables $Y, X_{1}, \ldots, X_{4}$ from a multivariate normal distribution. A mixed pattern of missingness is introduced, leaving an average of $72 \%$ of rows in each sample fully observed: $Y$ and $X_{4}$ are MCAR, while $X_{1}$ and $X_{2}$ are MAR as a function of $X_{3}$. We estimate the linear model $Y=\beta_{0}+\beta_{1} X_{1}+\beta_{2} X_{2}$ using four strategies: (1) MIDAS; (2) multivariate normal MI, implemented with the Amelia package in $\mathrm{R}$ (Honaker et al. 2011), which employs an expectation-maximization with bootstrapping algorithm; (3) listwise deletion, and (4) analysis of the complete dataset.10

Figure 3 plots the posterior densities of the estimated coefficients on $\beta_{0}, \beta_{1}$, and $\beta_{2}$ for each strategy. For all three parameters, MIDAS yields very similar results to Amelia. Both sets of estimates are close to the true density, though in the case of $\beta_{1}$ have smaller peaks and larger variances (due to their lower information content). Listwise deletion estimates, by contrast, are severely biased away the true density of every parameter, mostly possessing the incorrect sign as well as a higher variance than the other densities.

\footnotetext{
${ }^{10}$ In all performance tests conducted in this and the next section, $M=10$ for MI algorithms.
} 
FIGURE 3. Estimated Posterior Densities in MAR-1 Simulation Experiment

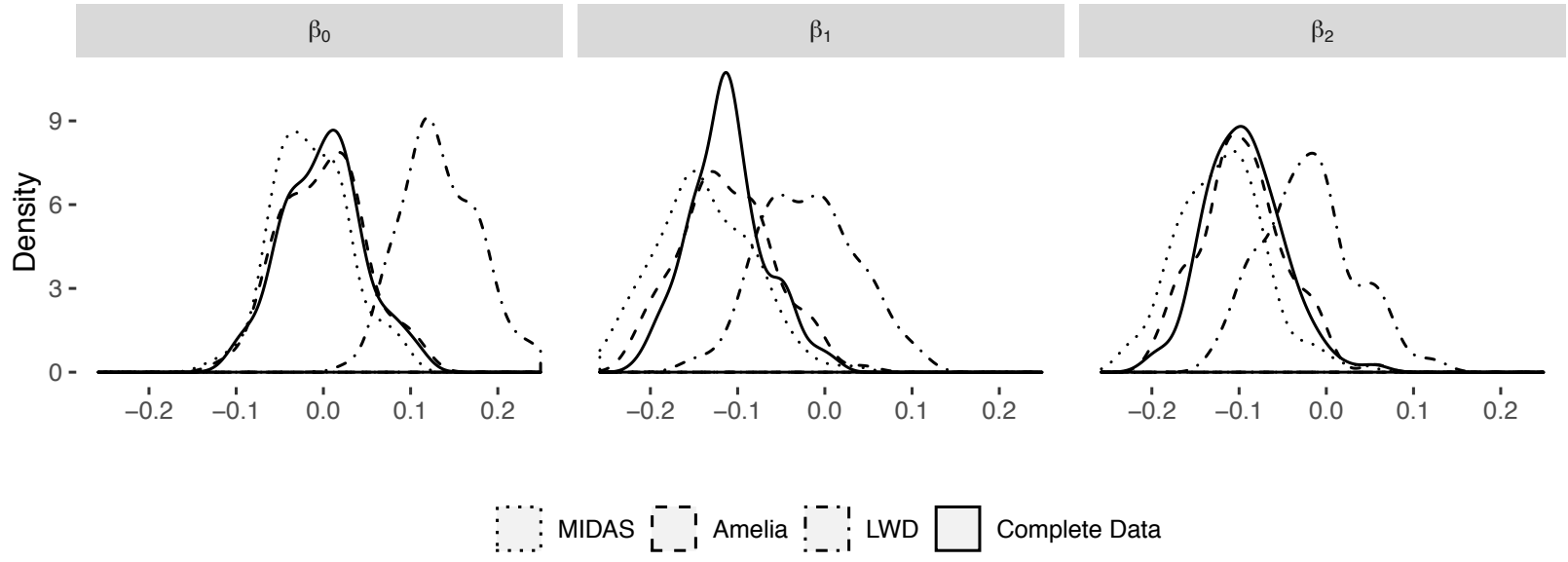

Coefficient estimates from a linear regression based on Monte Carlo-simulated data with 500 rows, $72 \%$ of which are fully observed, and five standardized variables drawn from a multivariate normal distribution.

The three strategies' estimated confidence intervals for the 100 simulations are plotted in Figure 4. Similarly to before, the Amelia and MIDAS intervals both exhibit good coverage for all three coefficients, encompassing the true estimate with a probability close to the ideal of 0.95 Listwise deletion's coverage is substantially worse in every case, excluding this estimate — and hence failing to appropriately capture uncertainty — in at least $40 \%$ of simulations for two of the three coefficients $\left(\beta_{0}\right.$ and $\left.\beta_{1}\right)$.12

\section{Simulation Test of Imputation and Linear Model Quality}

The continuous portion of Kropko et al.'s (2014) simulation-based accuracy test involves generating 1,000 multivariate normal datasets with 1,000 rows and eight standardized variables, and inducing MAR missingness in five of the latter (with proportions of $0.1,0.1$, $0.1,0.1$, and 0.25 ). To assess how the strength of relationships between variables affects MIDAS's performance, we generate two versions of the simulated datasets: one in which

\footnotetext{
${ }^{11}$ Amelia's coverage rates are marginally closer $\left(\beta_{0}=0.93, \beta_{1}=0.95, \beta_{2}=0.94\right)$ to the ideal than MIDAS's ( $\beta_{0}=0.93, \beta_{1}=0.86, \beta_{2}=0.87$ ), as should be expected under multivariate normal conditions.

${ }^{12}$ The coverage rates are $\beta_{0}=0.22, \beta_{1}=0.60, \beta_{2}=0.79$.
} 
FIGURE 4. Estimated 95\% Confidence Intervals in MAR-1 Simulation Experiment
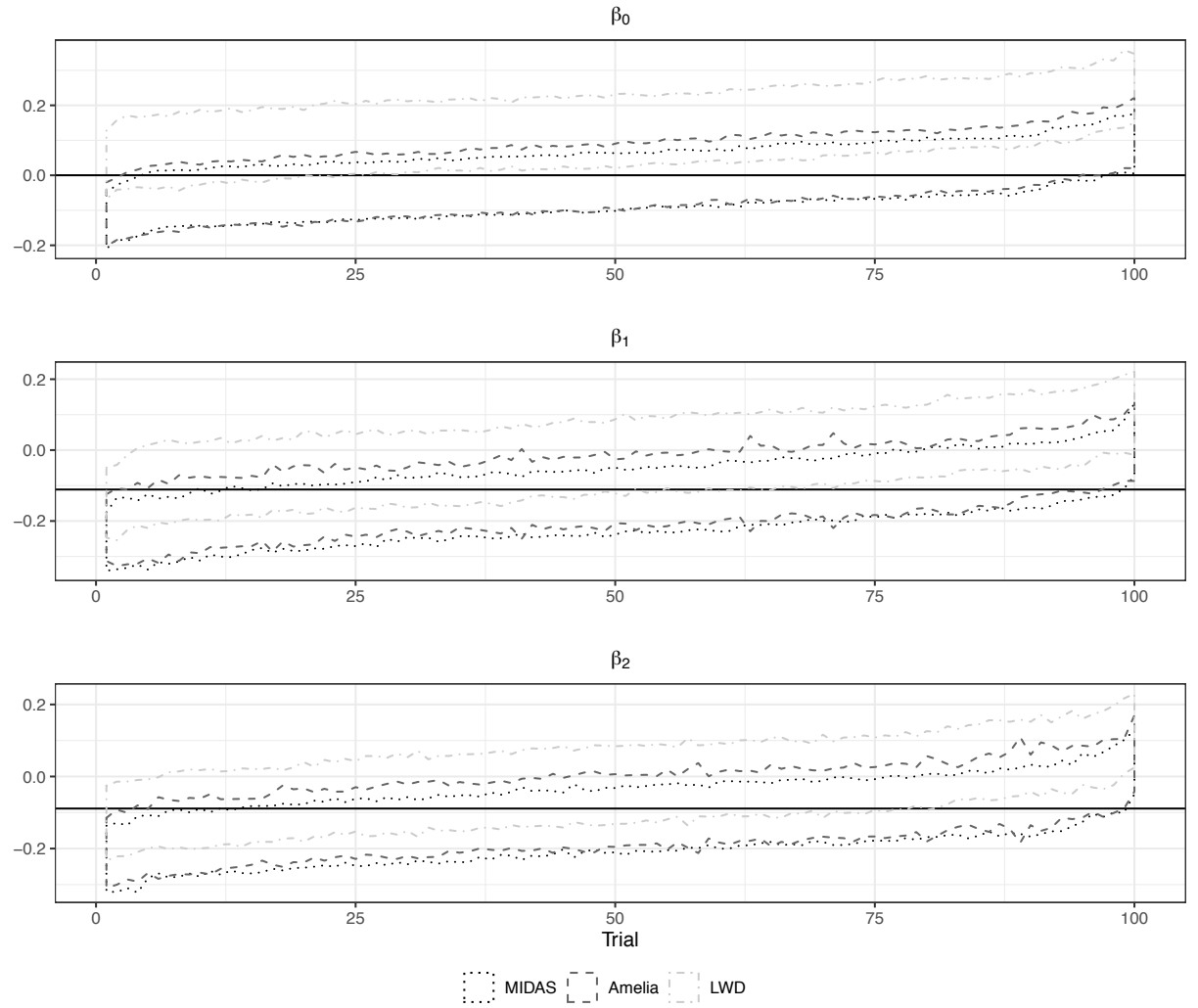

The distribution of each set of coefficient estimates is plotted in Figure 3. The solid black lines indicate full-data ("true") coefficients.

correlations between variables are moderate and another in which they are strong ${ }^{13}$

In addition to MIDAS, five missing-data strategies are applied to the incomplete datasets: (1) conditional MI, implemented with the mi package in $\mathrm{R}$ (Su et al. 2011); multivariate normal MI, implemented with (2) Amelia and (3) the norm package in R (which employs a traditional expectation-maximization algorithm) (Schafer and Olsen 1998); (4) listwise deletion; and (5) replacing missing values with draws from each variable's marginal distribution. The six strategies are assessed on two metrics: (1) RMSE relative to true values (averaging imputed values); and (2) the accuracy of coefficient estimates from a regression of one variable on the remaining seven, measured as (i) the Mahalanobis distance between

${ }^{13} \mathrm{As}$ Kropko et al. use the random data function rdata.frame in $\mathrm{R}$ to simulate the datasets, we model moderate and strong intercorrelations by setting the eta argument of this function to 300 and to 1000, respectively. 
FIGURE 5. Inverse Imputation and Linear Model Accuracy in Kropko et al. Simulation Test

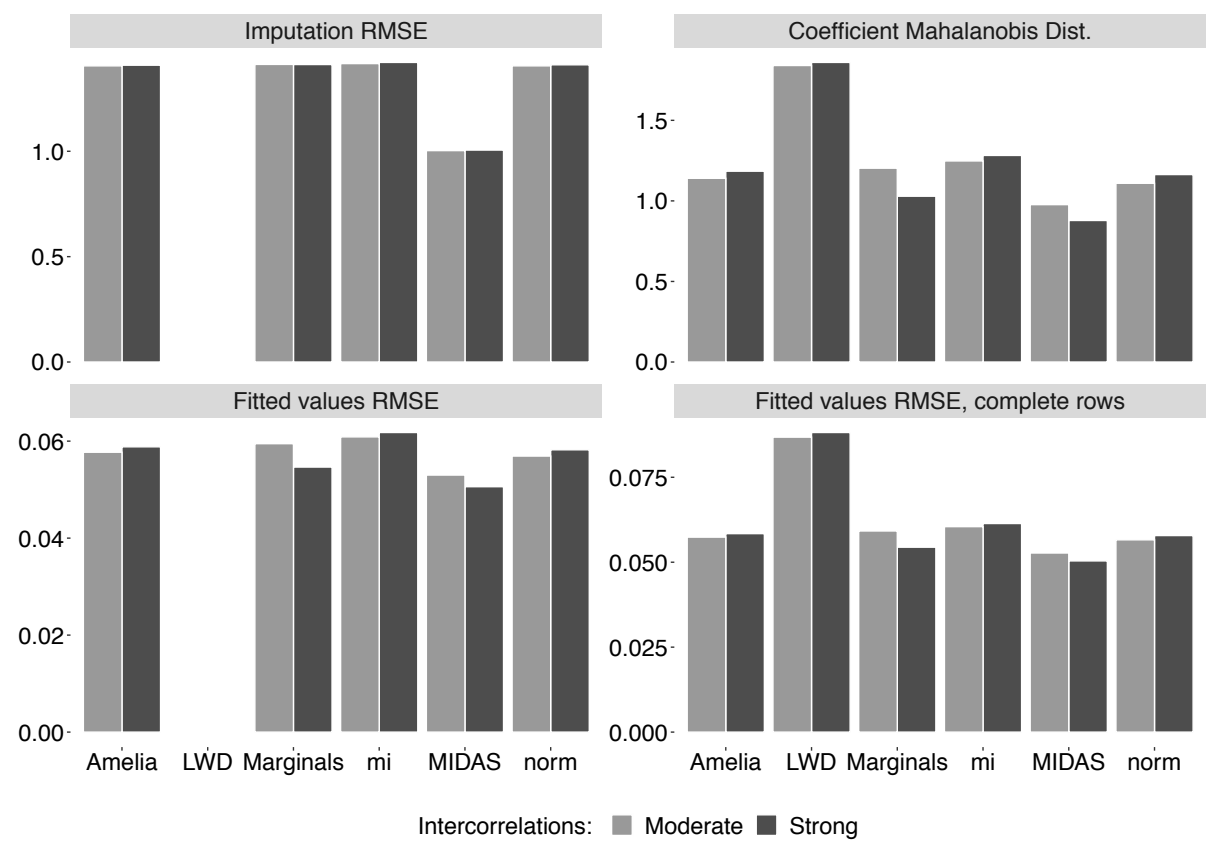

The results are based on Monte Carlo-simulated data with 1,000 rows and eight standardized variables, five of which contain MAR missingness, drawn from a multivariate normal distribution. Lower RMSE indicates greater imputation/fitted-value accuracy; lower Mahalanobis distances indicate greater coefficient accuracy.

model estimates and complete-data estimates, (ii) the RMSE of model fitted values relative to complete-data fitted values, and (iii) the previous metric excluding incomplete rows.

The results are displayed in Figure 5, In the moderate-correlation scenario, MIDAS outperforms the other four MI strategies on all four metrics. When we strengthen correlations, this performance gap remains essentially the same in terms of imputation accuracy but becomes even larger in terms of coefficient and fitted-value accuracy (except with respect to marginal draws). Even without a linearity constraint, therefore, MIDAS can produce accurate imputed values as well as minimally biased parameter estimates under multivariate normality, with its absolute and relative performance improving with the strength of relationships between variables. 


\section{Applied Test with Adult Dataset}

Real data, of course, are rarely multivariate normal. We thus supplement the previous simulation exercises with an applied accuracy test based on the Adult dataset, an extract from the 1994 United States Census that measures 15 characteristics of 48,842 individuals (a mixture of continuous and categorical variables). ${ }^{14}$ We select this dataset for two reasons. First, in addition to being frequently used by political scientists, it is a standard benchmarking dataset for machine learning tasks. Second, it is one of the few real social science datasets we were able to find that is almost entirely complete - just $0.009 \%$ of values are missing - which gives us near-complete discretion to manipulate missingness in the test (while mitigating possible concerns about the exclusion of originally missing values). Summary statistics for the dataset are provided in Appendix 4.

In contrast to the previous tests, we separately induce varying proportions of MCAR, MAR, and MNAR missingness in the dataset. For each missingness pattern, we create four versions of the dataset in which $30 \%, 50 \%, 70 \%$, and $90 \%$ of columns are randomly selected for corruption. In the MCAR treatment, half of the values in the selected columns are randomly set to missing. In the MAR treatment, a missingness indicator $L$ is randomly drawn from the non-selected columns. If $L$ is continuous, a subset of observations at or below its median value are set to missing in the selected columns; if $L$ is categorical, half of its categories are randomly sampled and a subset of corresponding observations in the selected columns are set to missing. The MNAR treatment is similar to the MAR treatment, with the key difference that $L$ is the selected column itself. These treatments are described in more detail in Appendix 4. Since Amelia's runtime substantially increases when categorical variables have more than 10 classes (which is prohibited in its default settings), native_country, occupation, and education are excluded from the corruption process.

\footnotetext{
${ }^{14}$ Available on the UC Irvine Machine Learning Repository: https://archive.ics.uci.edu/ml/datasets/adult.
} 
FIGURE 6. Results of Applied Imputation Accuracy Test

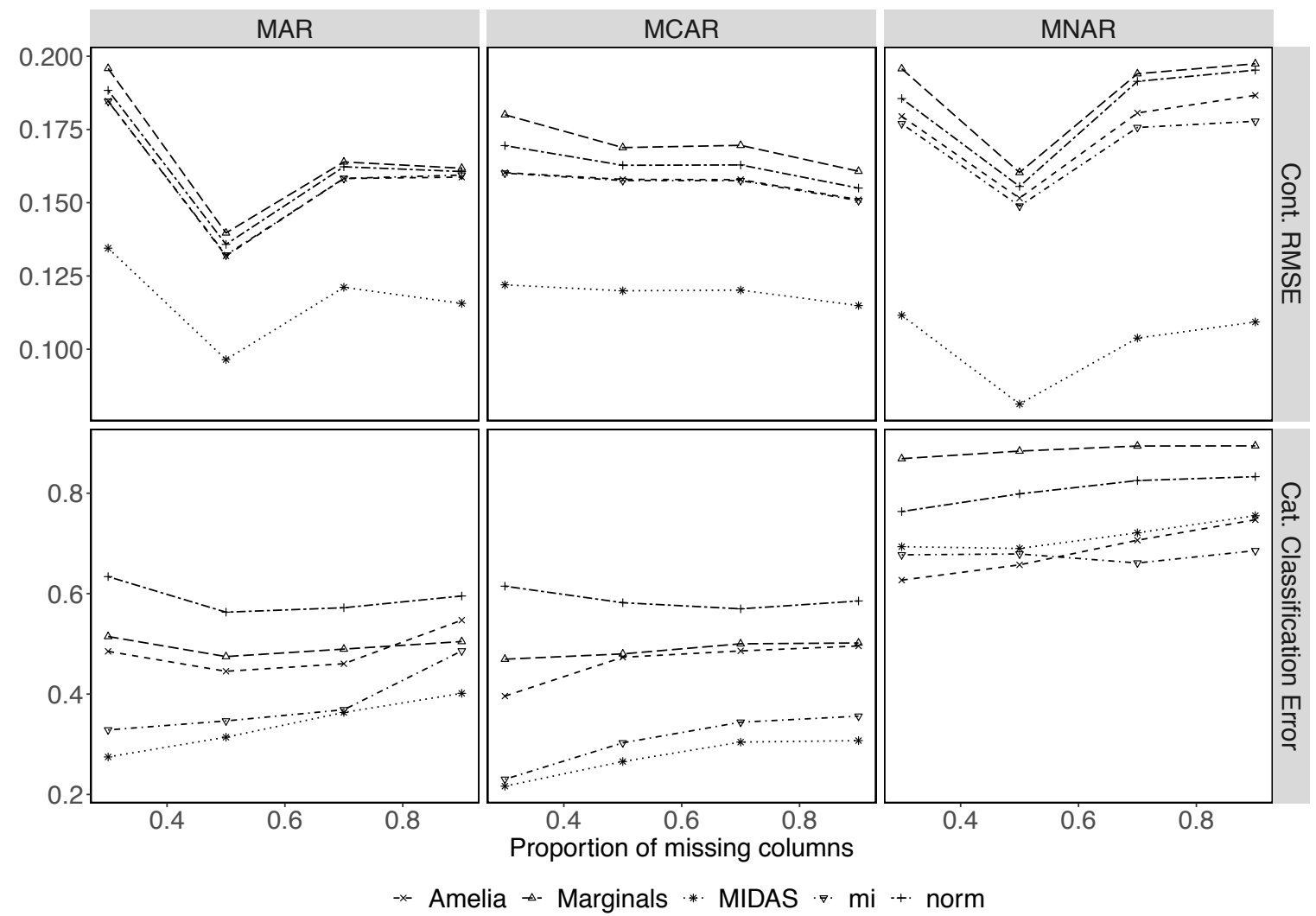

MCAR, MAR, and MNAR missingness are separately induced in varying proportions of randomly selected columns in the Adult dataset, with up to $50 \%$ of values are set as missing. Lower RMSE and classification error values indicate greater imputation accuracy.

We include the same five missing-data strategies as the previous test, comparing their imputation accuracy using similar metrics: the RMSE of imputed versus actual values for continuous variables; and classification error for categorical variables.15 We refrain from conducting a model-based accuracy test because, unlike in Kropko et al.'s simulation, we do not know the true joint distribution of the data. We instantiate MIDAS with two hidden layers of 256 nodes, an input corruption proportion of 0.75 , and 20 training epochs, leaving all other hyperparameters at their default settings. ${ }^{16}$ Amelia only converges with

${ }^{15}$ To ensure that differences in the strategies' treatment of categorical variables do not cause inconsistencies in the results, we round all imputed values for such variables to integers and bound them between 1 and the number of categories. Values that fall below (above) this range are assigned the first (last) category.

${ }^{16}$ The results of the test are robust to a range of alternative hyperparameter choices. 
a ridge prior of $1 \%$ of the number of rows in the imputation model, a modification that shrinks covariances between variables and thus introduces some level of bias (Honaker et al. 2011, 19-20). We swap the earlier version of the norm package, which is unable to handle the treated datasets, with an updated version based on the same algorithmic logic (Novo 2015). To enable mi to complete the test in a reasonable time, we modify its settings to generate imputed datasets after either 15 imputation iterations (default $=30$ ) or the default maximum iteration time of 20 minutes - whichever comes first.

The results are summarized in Figure 6. Across almost all corruption levels and missingness patterns, MIDAS's imputed values are more accurate than those of other strategies. This performance advantage is largest for continuous variables: the mean RMSE of MIDAS imputations is around 30\% lower than that of the next best algorithm, mi. The gap in classification accuracy is narrower but still clear in the MAR and MCAR scenarios. Under MNAR, Amelia and mi are the best category classifiers, though MIDAS's performance is comparable. In short, as we move to a more realistic setting in which multivariate normality does not hold, MIDAS continues to exhibit strong absolute and relative performance on key metrics of accuracy.

\section{Scalability Tests}

To facilitate comparison, the previous tests were conducted on small or medium-sized datasets that do not pose (major) computational problems for existing MI algorithms. We now relax this constraint, comparing the algorithms' efficiency in handling progressively larger datasets. We conduct separate tests for increasing numbers of columns and rows, though place greater weight on the former: additional columns are more computationally demanding for MI algorithms than additional rows because they entail a greater marginal 
increase in the number and complexity of relationships within the observed data. 17

\section{Column-Wise Scalability}

Rather than scaling up a purely simulated dataset, which is unlikely to capture the complexity and richness of real data, we conduct both tests using the 2018 Cooperative Congressional Election Study (CCES), a large-scale electoral survey commonly used by political scientists that encompasses a representative sample of 60,000 respondents in the United States. We focus on the subset of personal profile questions asked to all respondents, in addition to a selection of voting- and political activity-related questions (details are provided in Appendix (5). To generate a baseline sample for the column-wise test, we remove all columns that are perfectly collinear or that contain at least 10,000 missing values (which generally indicates structural missingness associated with survey flow) and all rows with responses of "don't know." This leaves a sample of 30,421 rows and 144 variables. Once categorical variables are one-hot encoded, there are 443 "effective" columns.

To examine the effect of increasing dataset width on imputation speed, we randomly draw columns without replacement from the baseline sample based on a target number of effective columns, which we vary from 25 to 400 . If, after selecting a given variable, the number of effective columns is more than $25 \%$ higher than the target, this variable is replaced and a new one is selected. After each dataset has been generated, we induce $50 \%$ MCAR missingness in every column. To ensure that the data do not become too sparse for imputation, we include the fully observed gender and birthyr variables in all samples.

We test the same five MI strategies as before, comparing the time they take to produce 10 completed datasets. MIDAS is instantiated with three hidden layers of 256 nodes, a dropout rate of 0.75 , and 30 training epochs - a relatively conservative setup, particularly

\footnotetext{
${ }^{17}$ Given the computational demands of these tests, we conducted them on an Amazon Web Services Linux m5.xlarge EC2 Instance virtual server (16 GB RAM, 4 vCPUs) running Ubuntu 18.04. A complete guide is included in the replication materials.
} 
FIGURE 7. Results of Column-Wise Scalability Test

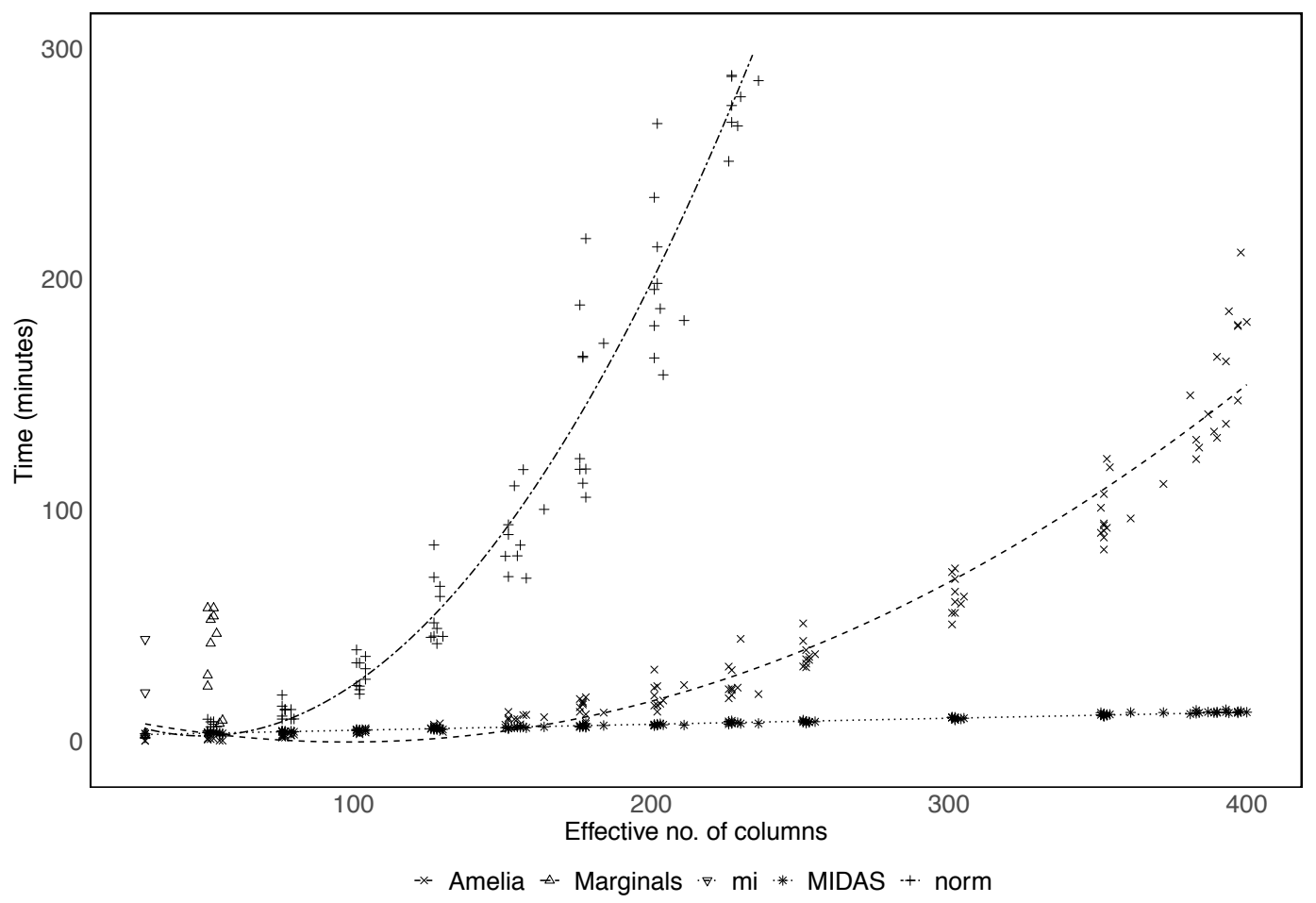

The $y$-axis measures the time taken to produce 10 completed versions of a CCES sample with 50 percent MCAR missingness; the $x$-axis measures the number of columns in the sample after categorical variables have been one-hot encoded. Points indicate runtimes; dashed lines show regression-based predicted values for each strategy.

for narrower datasets. Where possible, we parallelize the other MI algorithms using the doparallel and foreach packages in $\mathrm{R}$. If an algorithm requires bespoke formatting of the data prior to the imputation step, we include this in its total runtime. 18

Figure 7 displays the results, including predicted values from a regression of runtime on the effective number of columns (in which we add quadratic terms for Amelia and norm due to the distribution of their runtimes). Differences in scalability emerge even at the smallest widths, with the mi package and marginal draws recording runtimes several times longer than the remaining three algorithms for samples with 50 columns 19 The latter routines perform similarly up to this width, with Amelia slightly faster than Norm

\footnotetext{
${ }^{18}$ As before, we limit the number of imputation iterations to 15 per chain for mi.

${ }^{19}$ We therefore drop these two strategies for higher numbers of columns.
} 
and MIDAS but (unlike any other algorithm) failing to converge on several occasions. It is important to reiterate, moreover, that we do not adjust the MIDAS network's size by width; a three-layer, 256-node network is not necessary for narrow datasets, and a leaner architecture would result in faster computation.

As the number of columns increases, MIDAS's efficiency emerges clearly. MIDAS becomes faster than norm at a width of around 75 columns and Amelia just before 125 columns. By 200 columns, MIDAS is three times quicker than Amelia and almost 30 times quicker than norm. At the maximum number of columns in the test, 400, midas is 12 times faster than Amelia. Extrapolating from these results, Amelia would take more than 6000 hours to produce 10 completed versions of the full CCES, approximately 100 times longer than MIDAS. As indicated by the slope of the regression lines, MIDAS's efficiency advantage increases exponentially with the effective number of columns: the relationship between computation time and data width is linear for MIDAS but quadratic for Amelia and the other algorithms. This constitutes a major advantage in the Big Data era, in which datasets can contain thousands or even tens of thousands of variables.

\section{Row-Wise Scalability}

We test row-wise scalability by extracting a similar baseline sample from the CCES. To ensure a comparable overall runtime to the column-wise test, we focus on personal profile variables that are continuous, binary, or nominal and have fewer than seven levels. In total, the sample contains 22 variables and 34,441 complete rows. We vary sample length by bootstrapping rows to create datasets with between 5000 and 500,000 rows. We then induce MCAR missingness in 30\% of values in each column. As in the column-wise test, we exclude birthyr and gender from the missingness treatment to prevent excessive sparsity. As the baseline sample is smaller and less complex than before, we shrink the MIDAS network to two layers of 256 nodes and set the number of training epochs as 20 . 
FIGURE 8. Results of Row-Wise Scalability Test

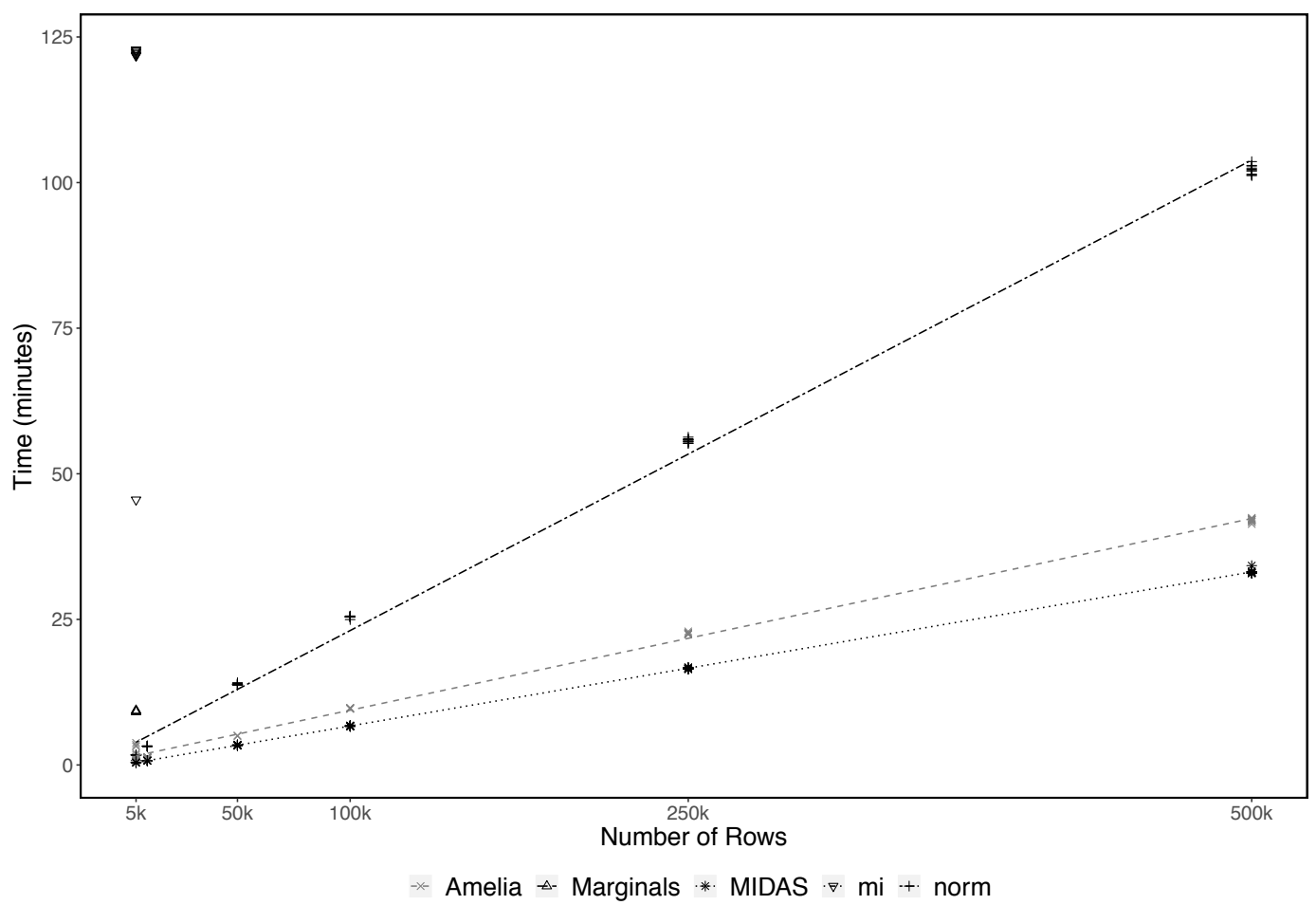

The $y$-axis measures the time taken to produce 10 completed versions of a CCES sample with 30 percent MCAR missingness; the $x$-axis measures the number of rows in the sample. Points indicate runtimes; dashed lines show regression-based predicted values for each strategy. Amelia's results are plotted in gray because it only converges with a ridge prior in the imputation model.

The results are plotted in Figure 8. Across all sample lengths, MIDAS is the most efficient strategy. For datasets with 500,000 rows, MIDAS's average runtime is three times quicker than than that of norm, the third fastest algorithm, and 25 percent quicker than that of Amelia, the second fastest, with these gaps increasing in proportion to length. Unlike in the column-wise test, therefore, computation time scales linearly with the number of rows for MIDAS as well as norm and Amelia, a finding consistent with the less intensive computational demands created by additional rows. As before, mi and marginal draws record the longest runtimes, producing the completed datasets in an average of 115 minutes and 9.2 minutes, respectively, at the smallest number of rows (5000) 20

\footnotetext{
${ }^{20}$ We again exclude these two strategies for longer samples.
} 
Note that while the performance gap between MIDAS and Amelia is smaller in this test, there are caveats to the latter's results. Amelia did not converge with any dataset without the inclusion of a bias-inducing ridge prior in the imputation model (of 0.005 times the number of rows), most likely due to high correlations among some variables. Even with this modification, it failed to converge in 16 of the 60 iterations of the simulation.

In both tests, therefore, MIDAS exhibits greater scalability than a host of widely used existing MI strategies, demonstrating an efficiency in handling increasing numbers of rows and, in particular, columns that render it well suited to Big Data applications.

\section{Applied Illustration: Estimating Ideology from CCES Data}

In this section, we provide a brief illustration of MIDAS's capacity to handle real missingdata situations whose scale presents computational difficulties for existing MI algorithms. We continue to focus on the CCES, whose large number of columns - a feature shared with other electoral surveys - can prevent the usage of such algorithms. Specifically, we use MIDAS to shed new light on the distribution of political ideology among respondents, a topic of substantive interest to scholars of electoral politics in the US and elsewhere.

Respondents to the CCES are asked to report their ideological position on a sevenpoint scale ranging from 1 for "Very Liberal" to 7 for "Very Conservative." Self-reported ideology, however, is known to be a noisy proxy for underlying beliefs (for instance, due to social desirability biases and variation in ideological positions across policy dimensions). A variety of approaches have been proposed to capture respondents' latent ideology, most of which involve estimating ideology using responses to policy-related questions. In the 2018 CCES, individuals are asked their opinion on a series of policy proposals in areas such as the budget, healthcare, and environmental protection. These items have a higher rate of nonresponse than the CCES in general, with an average of $14 \%$ of respondents failing to 
provide an answer. Does this missingness affect estimates of latent ideology?

Building on recent work by Ramseyer and Rasmussen (2016), we regress respondent $i$ 's self-reported ideology on responses to 19 policy questions in the CCES (see Appendix 6A for the list):

$$
\text { Self-Reported Ideology }_{i}=\alpha+\sum_{j=1}^{19} \beta_{j} \times \text { Policy }_{i, j}+\epsilon_{i}
$$

where $j$ denotes a given policy question. The fitted values from Equation 8 represent estimates of latent ideology. We compare such estimates under two missing-data strategies: (1) listwise deletion (following Ramseyer and Rasmussen), which risks bias because missingness is plausibly related to the outcome variable; and (2) MIDAS, which we implement using a rich battery of 163 demographic and socioeconomic variables - an imputation model too large to be computed by any existing MI algorithm - and a two-layer, 256node network trained for 200 epochs. ${ }^{21}$ MIDAS allows us to produce estimates for more than 10,000 more respondents, almost one-fifth of the full CCES.

Figure 9 plots the kernel densities of the two sets of latent ideology estimates. The two distributions have similar variances but divergent peaks; the null hypothesis that they are samples from the same distribution can be rejected at the $0.1 \%$ level under a KolmogorovSmirnov test. The distribution of listwise deletion estimates is skewed toward the left (liberal) side of the ideology scale - the modal estimate is $2-$ though also contains smaller peaks in the centre and on the right (conservative) side. The MIDAS estimates, in contrast, follow a more normal shape, peaking at 4. In the absence of imputation, therefore, there is a danger that analysts could substantially overestimate the proportion of strong liberal and strong conservative respondents in the sample.

\footnotetext{
${ }^{21}$ Some existing MI algorithms, such as Amelia, can accommodate subsets of the MIDAS imputation model. These subsets, however, exclude variables that are likely to be strong predictors of missingness in the policy items (e.g., state of residence in the case of Amelia). Consequently, as shown in Appendix 6B, the resulting latent ideology estimates are substantially closer to those produced by listwise deletion.
} 
FIGURE 9. Regression-Based Estimates of CCES Respondents' Latent Ideology

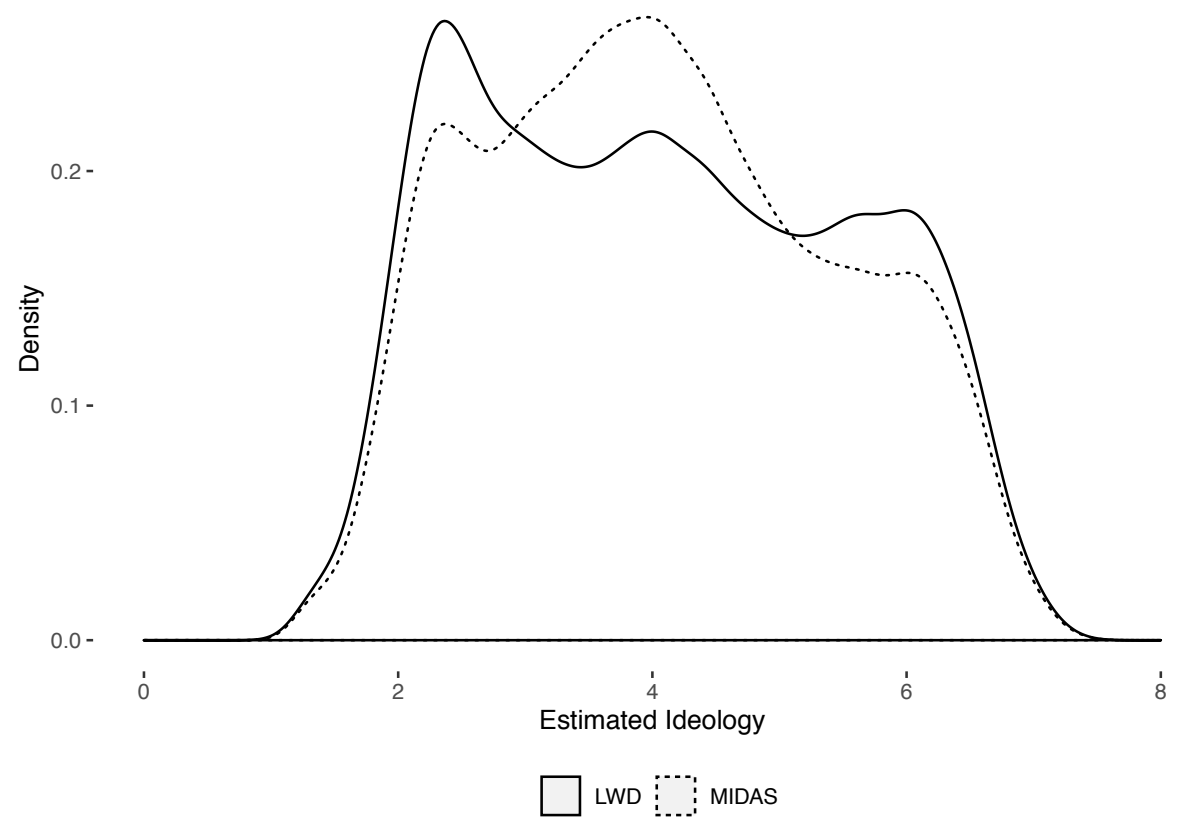

This finding also has implications for our understanding of the relationship between ideology and other variables of substantive interest in the CCES, for instance, respondents' assessment of President Donald Trump's performance in office. Table 1 summarizes the results of regressing responses to the CCES presidential job approval question, which range from 1 for "strongly approve" to 4 for "strongly disapprove," on (1) self-reported ideology and (2) the MIDAS-based regression estimates of latent ideology:22

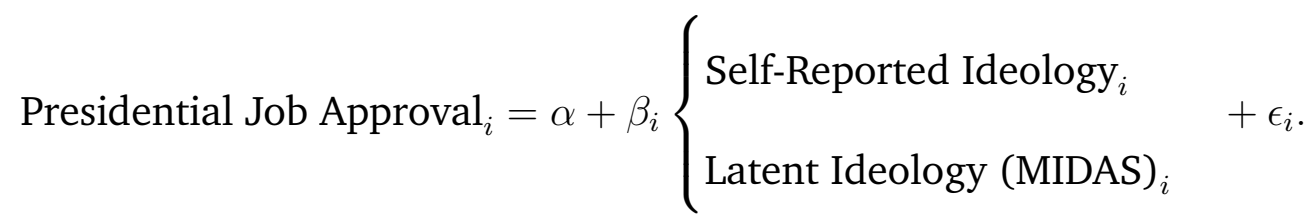

In both models, the estimated coefficient on the measure of ideology is negative and statistically significant, indicating that respondents classified as more liberal express lower average levels of presidential job approval. The MIDAS-based measure, however, is a far

${ }^{22}$ We remove "not sure" responses from the job approval variable for the self-reported model. These values are imputed in the MIDAS model. 
TABLE 1. Regression of Presidential Job Approval on Different Measures of Ideology

\begin{tabular}{lcccc}
\hline Measure of Ideology & $\boldsymbol{\beta}$ & Std. Error & Adj. $\boldsymbol{R}^{\mathbf{2}}$ & $\mathbf{~ N}$ \\
\hline Self-reported & -0.475 & 0.002 & 0.506 & 48713 \\
Regression-based (MIDAS) & -0.697 & 0.002 & 0.616 & 60000 \\
\hline
\end{tabular}

better predictor of job approval than the self-reported alternative, possessing a coefficient estimate almost 50\% larger (with an identical standard error) and accounting for $20 \%$ more model-adjusted variance in the outcome. In substantive terms, the MIDAS model's estimate implies that shifting from the most liberal to the most conservative respondent raises job approval by the full range of this variable's scale - a sizable increase.

In sum, by leveraging a wealth of contextual information on respondents, MIDAS helps to avoid nonresponse-related biases in the estimation of latent ideology, altering our understanding of this variable's distribution and relationship to presidential job approval.

\section{What Can Go Wrong?}

While MIDAS's flexibility render it suitable for a wide range of missing-data problems, there are nevertheless circumstances in which it may perform suboptimally. First, MIDAS cannot, of course, avoid bias when the usual assumptions of MI are violated: data are MNAR, the posited distribution of the data is a poor approximation to reality, or the imputation model is misspecified in some other way. However, as noted earlier - and demonstrated in the applied accuracy test - MIDAS can still perform relatively well under MNAR when there are strong predictors of missingness in the imputation model.

Second, like other approaches to MI, MIDAS is not guaranteed to perform well with certain unconventional data structures, such as non-exchangable data, multilevel data, survival data, and spatially lagged data (Lall 2016). In general, however, we have found MIDAS to be surprisingly effective at learning observed-data relationships within these structures (without the inclusion of any special features in the imputation model). Appendix 7 
provides an illustration of this capacity in the context of time-series cross-sectional data perhaps the most common form of non-exchangable data in applied social science research - adapting an exercise conducted by Honaker and King (2010) to show how MIDAS can impute smooth nonlinear time trends in economic data. This illustration, which involves another dataset too large to be processed in its entirety by existing MI algorithms, highlights the way in which the adaptability of neural networks can sometimes mitigate the need for manual variable selection and feature transformation.

Third, a well-known limitation of variational approximation methods is their tendency to underestimate the variance of the posterior density (Grimmer 2010). In the case of MIDAS, this is most likely to occur when data are concentrated in particular regions of the joint probability space - in other words, when the dataset contains many very similar input-output pairs - which can result in an approximation that only maps a small subset of this space. It should be noted, however, that the inferential consequences of posterior variance underestimation continue to be debated, and some studies find that it may not seriously compromise the accuracy of estimated posterior densities (e.g., Blei and Jordan 2006; Braun and McAuliffe 2010). Indeed, the MAR-1 experiment shows that MIDAS can yield approximately correct posteriors even under unfavorable statistical conditions.

Finally, MIDAS inherits the general risks associated with neural network-based methods. These include misspecification of hyperparameters (in particular network structure, activation functions, and the number of training epochs), which can result in bias; overfitting — despite MIDAS's heavy inbuilt regularization — the likelihood of which increases with the size, dimensionality, and sparsity of the dataset; and poor performance on very small datasets. Such risks can be compounded by the "black box" nature of neural networks, which makes it difficult for analysts to conduct parameter and posterior checks to identify problems. Although the midas class offers a variety of diagnostic tools to help analysts conduct such checks (discussed in Appendix 2), we fully acknowledge that they 
do not guarantee detection of all problems.

\section{Concluding Remarks}

As the scale and complexity of political science data continue to grow, it is increasingly important that analysts have access to accurate, fast, and scalable methods for dealing with missing values. The approach we have developed in this study, MIDAS, offers a way of efficiently multiply imputing missing values in large and complex datasets with high levels of accuracy. A battery of tests involving simulated and real data have provided evidence of these advantages - both absolute and relative to existing MI strategies across a range of settings.

To be sure, MIDAS is not a panacea for missing-data problems in the emerging era of Big Data in political science. As discussed earlier, despite its flexibility, the approach is not guaranteed to perform well with every type of data and may be not be straightforward to optimize for particular applications. Nevertheless, we believe that it constitutes a useful addition to the methodological toolkit of applied researchers and nicely complements the strengths of existing approaches to MI. Indeed, it is precisely the kinds of applications with which these approaches can struggle where MIDAS comes into its own.

In bringing together MI, DAs, dropout, and other methods, this study contributes to the influential political science literature on missing data as well as to promising emerging research agendas on deep learning, neural networks, Big Data, variational inference, and Bayesian modeling in the discipline. In addition, given the close connection between missing-data problems and the estimation of treatment effects, we believe that MIDAS may have implications for the study of causal inference - and perhaps even practical uses in this area, for instance, as a tool for mitigating measurement error (Blackwell et al. 2017) or imputing counterfactuals for treated units within a potential outcomes framework ( $\mathrm{Xu}$ 
2017). In this respect, it could facilitate ongoing efforts to make Big Data, machine learning, and causal inference "work together" to generate new substantive knowledge about politics (Grimmer 2015).

\section{References}

Beaulieu-Jones, Brett K. and Casey Greene (2016). Semi-supervised learning of the electronic health record for phenotype stratification. Journal of Biomedical Informatics 64 (2), 168-178.

Blackwell, Matthew , James Honaker, and Gary King (2017). A unified approach to measurement error and missing data: Overview and applications. Sociological Methods \& Research 46 (3), 303-341.

Blei, David M. and Michael I. Jordan (2006). Variational inference for dirichlet process mixtures. Bayesian Analysis 1 (1), 121-143.

Braun, Michael and Jon McAuliffe (2010). Variational inference for large-scale models of discrete choice. Journal of the American Statistical Association 105 (489), 324-335.

Cranmer, Skyler J. and Jeff Gill (2013). We have to be discrete about this: A nonparametric imputation technique for missing categorical data. British Journal of Political Science 43 (2), 425-449.

Duan, Yanjie , Yisheng Lv, Wenwen Kang, and Yifei Zhao (2014). A deep learning based approach for traffic data imputation. In 17th International IEEE Conference on Intelligent Transportation Systems (ITSC), pp. 912-917. IEEE.

Gal, Yarin and Zoubin Ghahramani (2016). Dropout as a bayesian approximation: Rep- 
resenting model uncertainty in deep learning. In Proceedings of the 33rd International Conference on Machine Learning, pp. 1050-1059. ACM.

Glorot, Xavier and Yoshua Bengio (2010). Understanding the difficulty of training deep feedforward neural networks. In Proceedings of the 13th International Conference on Artificial Intelligence and Statistics, pp. 249-256. ICAIS.

Gondara, Lovedeep and Ke Wang (2018). Mida: Multiple imputation using denoising autoencoders. In Pacific-Asia Conference on Knowledge Discovery and Data Mining: Advances in Knowledge Discovery and Data Mining, pp. 260-272. Springer, Cham.

Goodfellow, Ian , Yoshua Bengio, and Aaron Courville (2016). Deep Learning. Cambridge, MA: The MIT Press.

Grimmer, Justin (2010). An introduction to bayesian inference via variational approximations. Political Analysis 19 (1), 32-47.

Grimmer, Justin (2015). We are all social scientists now: How big data, machine learning, and causal inference work together. PS: Political Science \& Politics 48(1), 80-83.

Hinton, Geoffrey E. , Nitish Srivastava, Alex Krizhevsky, Ilya Sutskever, and Ruslan R. Salakhutdinov (2012). Improving neural networks by preventing co-adaptation of feature detectors. Neural Networks 2, 1-18.

Honaker, James and Gary King (2010). What to do about missing values in time-series cross-section data. American Journal of Political Science 54 (2), 561-581.

Honaker, James , Gary King, and Matthew Blackwell (2011). Amelia II: A program for missing data. Journal of Statistical Software 45 (7), 1-47. 
King, Gary , James Honaker, Anne Joseph, and Kenneth Scheve (2001). Analyzing incomplete political science data: An alternative algorithm for multiple imputation. American Political Science Review 95 (1), 49-69.

Kingma, Diederik P. and Jimmy Ba (2015). Adam: A method for stochastic optimization. In 3rd International Conference on Learning Representations (ICLR), Conference Track Proceedings. ICLR.

Kropko, Jonathan, Ben Goodrich, Andrew Gelman, and Jennifer Hill (2014). Multiple imputation for continuous and categorical data: Comparing joint multivariate normal and conditional approaches. Political Analysis 22 (4), 497-219.

Lall, Ranjit (2016). How multiple imputation makes a difference. Political Analysis 24 (4), 414-433.

Little, Roderick J.A. and Donald Rubin (2002). Statistical Analysis with Missing Data (Second Edition). Hoboken, NJ: Wiley.

Mustillo, Sarah and Soyoung Kwon (2015). Auxiliary variables in multiple imputation when data are missing not at random. The Journal of Mathematical Sociology 39 (2), 73-91.

Novo, Alvaro A. (2015). norm. Vienna, Austria: R Foundation for Statistical Computing.

Ramseyer, J. Mark and Eric B. Rasmussen (2016). Voter ideology: Regression measurement of position on the left-right spectrum.

Rubin, Donald B. (1987). Multiple Imputation for Nonresponse in Surveys. New York, NY: John Wiley \& Sons. 
Rumelhart, David E. , Geoffrey E. Hinton, and Ronald J. Williams (1986a). Learning internal representations by error propagation. In D. E. Rumelhart and J. L. McClelland (Eds.), Parallel Distributed Processing (Volume 1), pp. 318-362. Cambridge, MA: MIT Press.

Rumelhart, David E. , Geoffrey E. Hinton, and Ronald J. Williams (1986b). Learning representations by back-propagating errors. Nature 323 (6088), 533-536.

Schafer, Joseph L. and Maren K. Olsen (1998). Multiple imputation for multivariate missing-data problems: A data analyst's perspective. Multivariate Behavioral Research 33 (4), 545-571.

Su, Yu-Sung , Andrew Gelman, Jennifer Hill, and Masanao Yajima (2011). Multiple imputation with diagnostics (mi) in r: Opening windows into the black box. Journal of Statistical Software 45 (2), 1-31.

van Buuren, Stef (2012). Flexible Imputation of Missing Data. Boca Raton: Taylor and Francis.

Vincent, Pascal, Hugo Larochelle, Yoshua Bengio, and Pierre-Antoine Manzagol (2008). Extracting and composing robust features with denoising autoencoders. In Proceedings of the 25th International Conference on Machine Learning, pp. 1096-1103. ACM.

Xu, Yiqing (2017). Generalized synthetic control method: Causal inference with interactive fixed effects models. Political Analysis 25(1), 57-76. 


\section{Appendices for "Applying the MIDAS Touch: How to Handle Missing Values in Large and Complex Data"}

April 2, 2020

\section{Contents}

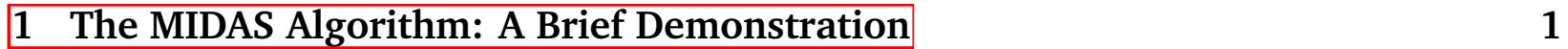

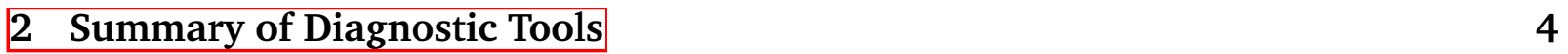

3 Technical Details on MIDAS Model and Algorithm 6

$3 \mathrm{~A}$ Objective Function $\ldots \ldots \ldots \ldots$

3B Training Steps . . . . . . . . . . . . . . . . 7

4 Additional Information on Applied Accuracy Test 10

5 Additional Information on Scalability Analysis 12

5 A List of Variables in Column-Wise Test . . . . . . . . . . . . . . . . . . . 12

5B List of Variables in Row-Wise Test . . . . . . . . . . . . . . . . 13

6 Additional Information on Latent Ideology Estimation 13

6 A List of Policy Questions . . . . . . . . . . . . . . . . . . . 13

6B Comparison with Amelia . . . . . . . . . . . . . 13

7 Imputing Time-Series Cross-Sectional Data: An Illustration 16 


\section{The MIDAS Algorithm: A Brief Demonstration}

This appendix provides a brief demonstration of the midas class in the Python programming environment, the software we have developed to implement MIDAS. We show how to use the class to multiply impute missing values in the Adult census dataset, the basis for our applied accuracy test.

To access the class, users must have TensorFlow installed as a pip package in their Python environment. MIDAS is written in TensorFlow 1.X API; users of TensorFlow 2.X can install the correct version via the command line:

pip install tensorflow==1.14.0

We recommend creating a Conda environment before reinstalling TensorFlow to avoid conflicts with projects using later versions of the package.

Users should then place the files midas.py and adult.csv in their working Python directory. Both files can be obtained from the MIDAS GitHub page [hyperlink to be inserted]. The midas class and Adult dataset can then be imported:

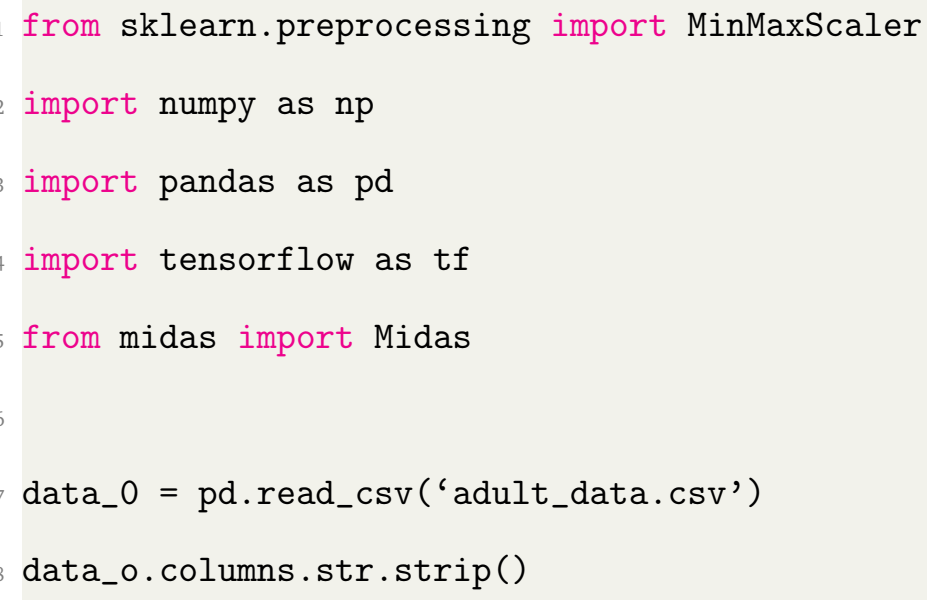

As the dataset has a very low proportion of missingness (one of the reasons we selected it for the accuracy test), we randomly set 5000 observed values as missing in each column:17

${ }^{1}$ This procedure differs from the more complex missingness treatments in our applied accuracy test (which we implement in $\mathrm{R}$ because the various MI algorithms require bespoke data formatting). 


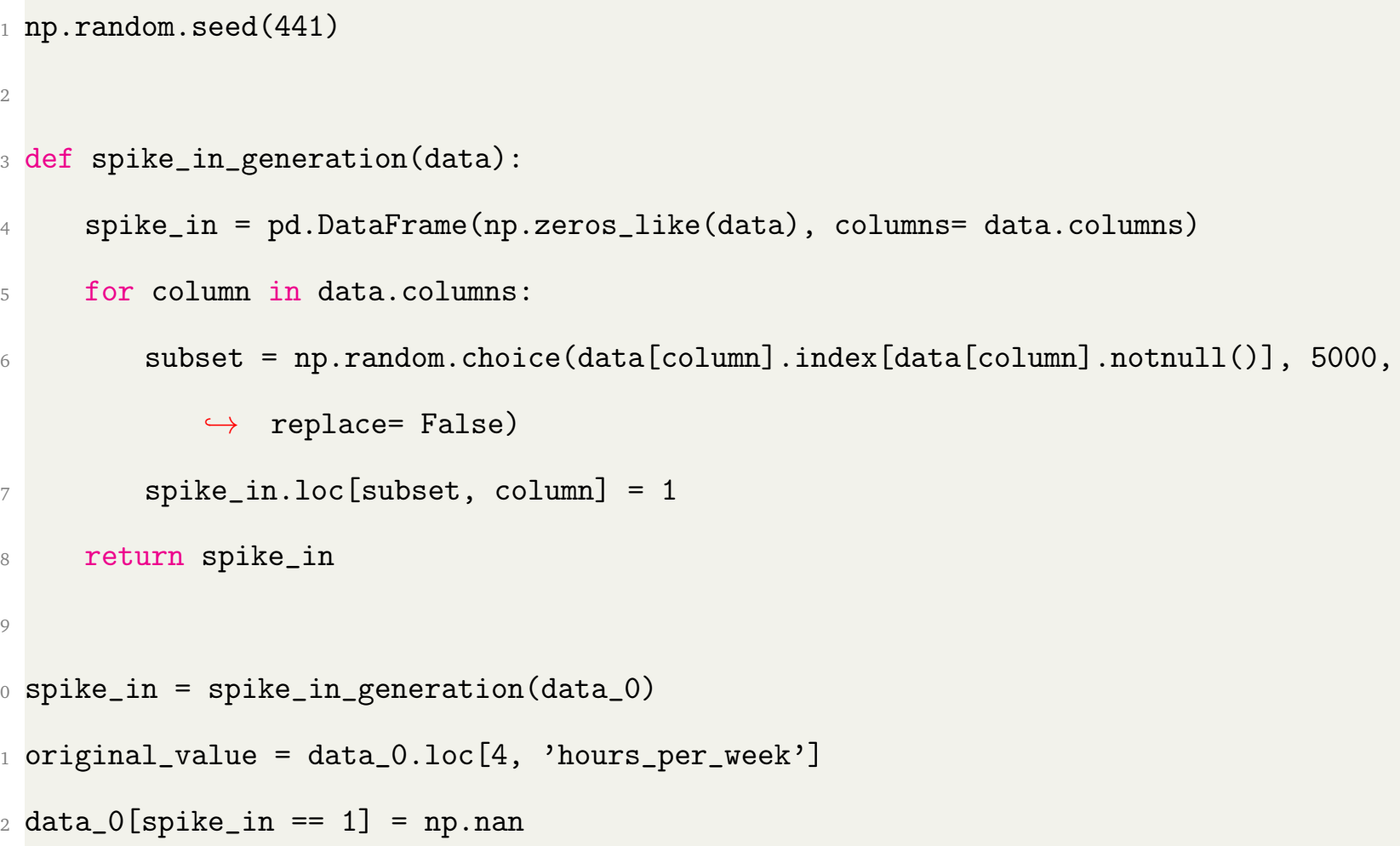

Next, we list categorical variables in a vector and one-hot encode them using an inbuilt function in the pandas package:

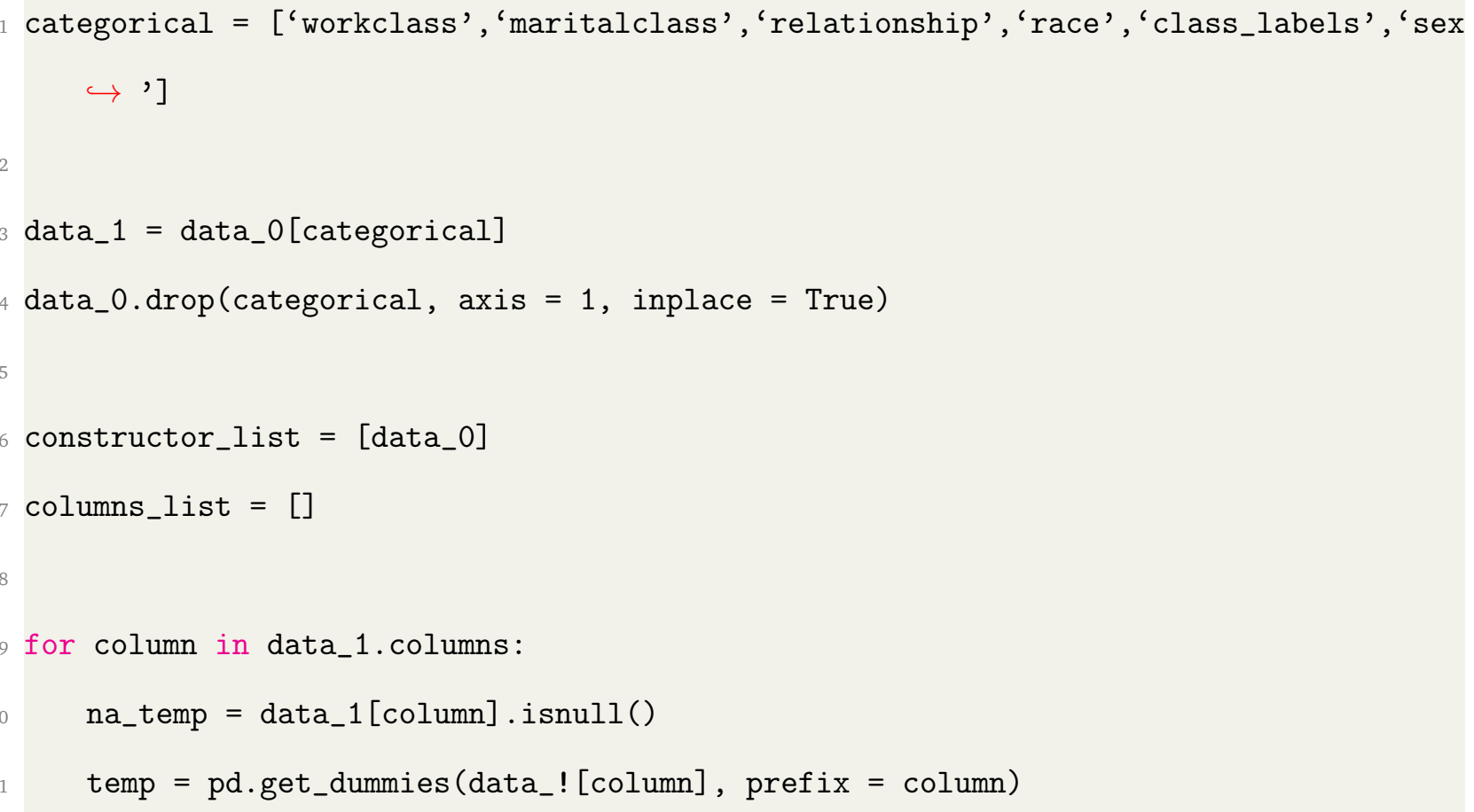




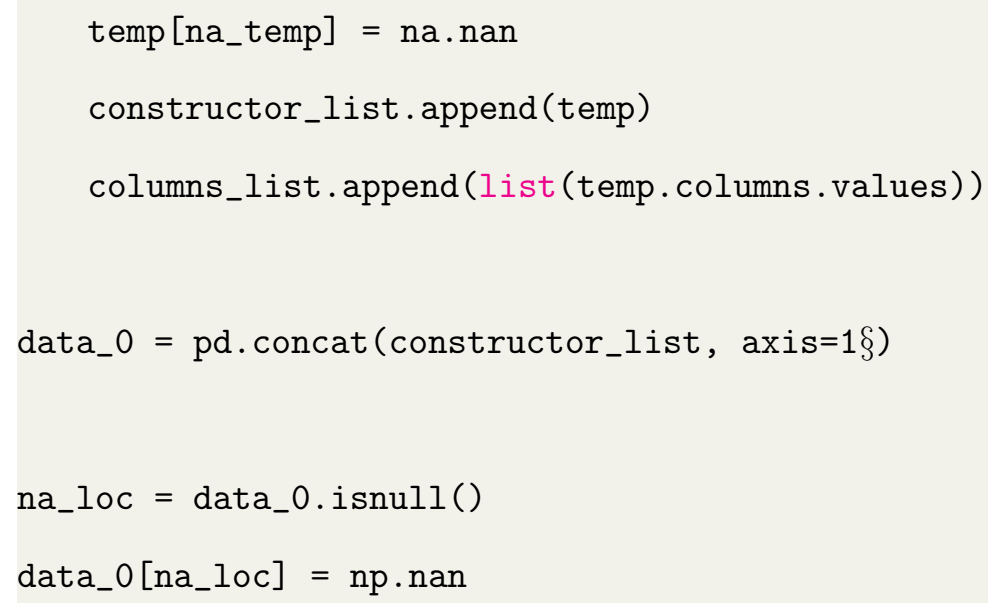

The data are now ready to be fed into the midas algorithm, which involves three steps. First, we specify the dimensions, input corruption proportion, and other hyperparameters of the MIDAS neural network. Second, we build a MIDAS model based on the data. The vector of one-hot-encoded column names should be passed to the softmax_columns argument, as MIDAS employs a softmax final-layer activation function for categorical variables. Third, we train the model on the data, setting the number of training epochs as 20 in this example:

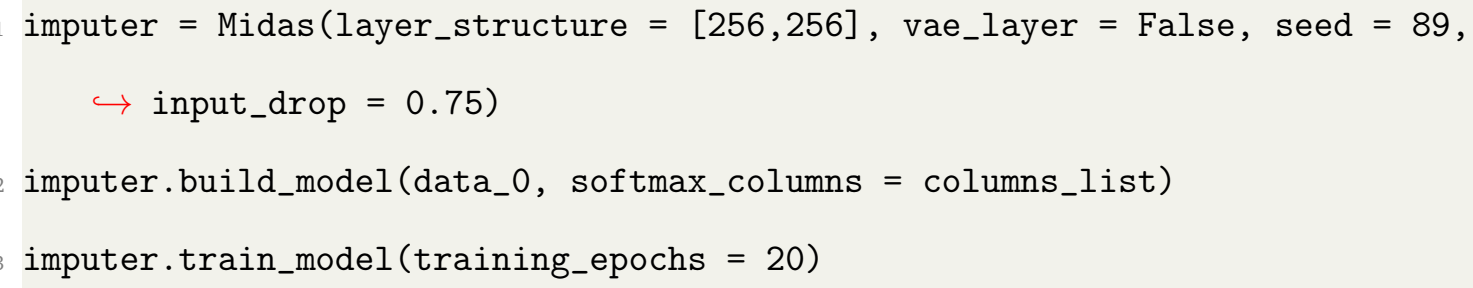

Once training is complete, we can generate any number of imputed datasets using the generate_samples function (here we set $M$ as 10). Users can then either write these imputations to separate .csv files or work with them directly in Python:

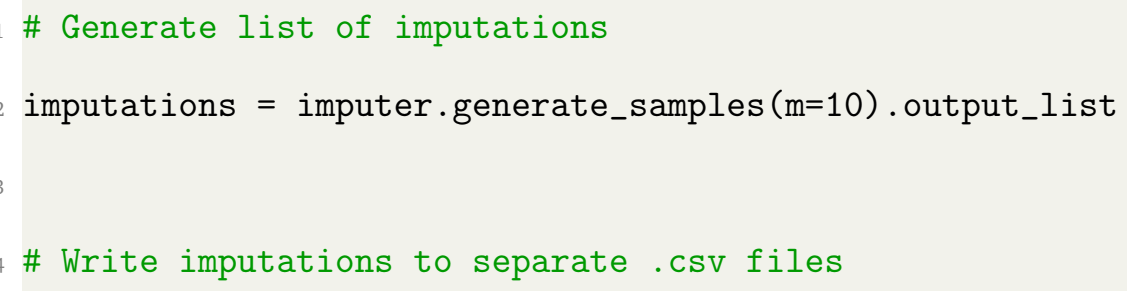




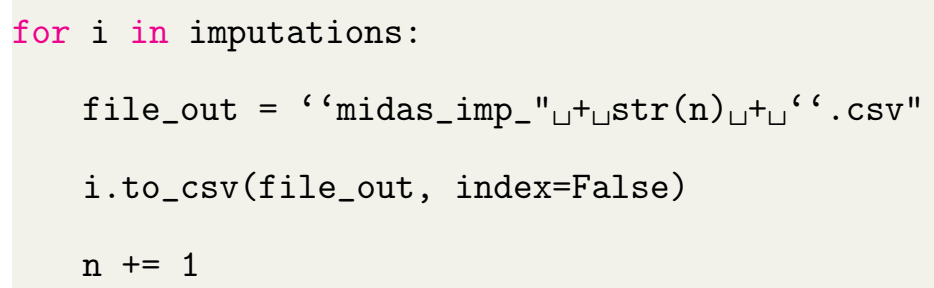

\section{Summary of Diagnostic Tools}

The performance of modern machine learning techniques depends heavily on the length of training — which affects the risk of overfitting — and the choice of model hyperparameters (Probst et al. 2019). To help users of MIDAS assess the fit of the imputation model and calibrate hyperparameters, we provide two diagnostic tools. The first is the technique of “overimputation" (Blackwell et al. 2017; Honaker et al. 2011). This involves sequentially removing observed values from the dataset, generating a large number of imputations for each value, and checking the accuracy of these imputations. Accuracy is measured with (1) the RMSE of imputed values versus true values for continuous variables and (2) classification error for categorical variables. To ensure a good fit, we recommend selecting the number of training epochs that minimizes the average value of these metrics (weighted by the proportion of continuous versus categorical variables). By reducing the risk of overtraining, this "early stopping" rule effectively serves as an extra layer of regularization in a MIDAS network.

In the midas class, overimputation can be implemented using the overimpute function (described in more detail on the MIDAS GitHub page). This function plots values of the RMSE and classification error metrics for each training epoch. Initially, these values should decline with additional epochs as the MIDAS network learns increasingly accurate approximations of the missing-data posterior. As suggested above, if and when error begins to rise, the number of epochs specified in the train.model function (demonstrated in 
Appendix 11) should be capped before this point. The plot_all argument of overimpute compares the distribution of overimputed versus original values, allowing users to visually inspect whether the former fall within a reasonable range (implying a good model fit). The default hyperparameter settings for overimpute are a corruption proportion (spikein) of 0.1 and 100 training epochs (training_epochs).

The second diagnostic tool is the generation of entirely new observations using a variational autoencoder component. Variational autoencoders are another extension of the classical autoencoder that encode inputs not to a fixed vector $\mathrm{z}$ but to a distribution over the latent space $p(\mathbf{z})$ (Kingma and Welling 2013; Rezende et al. 2014). The loss function minimized during training includes a regularization term (in addition to the usual reconstruction term) that constrains the latent distribution to approximate normality, reducing the risk of an irregular latent space in which similar data points can become very different after decoding. Samples from the latent distribution $\mathbf{z} \sim p(\mathbf{z} \mid \mathbf{x})$ will thus tend to more closely follow the input density than a regular (deterministic) latent representation $\mathrm{z}$, rendering them better suited to the task of generative modeling.

In the midas class, the variational autoencoder component can be activated by setting vae_layer $=$ True in the Midas function. This inserts a variational autoencoder layer after the denoising portion of a MIDAS network, which probabilistically maps inputs to a latent distribution in the manner described above. After training, samples are drawn from this distribution and decoded to produce new observations. In general, the greater the similarity between these observations and the input data, the better the fit of the imputation model. Default settings for vae_layer hyperparameters - which include the number of normal clusters assumed to characterize the input data (latent_space_size), the variance of these distributions (vae_sample_var), and the strength of our normal prior (vae_alpha) - follow standard conventions in autoencoder applications.

We favor overimputation and data generation over customary train/test split approaches 
to model validation for two reasons. First, the latter have been found to systematically underestimate error in autoencoders and other unsupervised methods of nonlinear dimensionality reduction where there is no clear target value (Christiansen 2005; Scholz 2012). Second, they prevent us from training the MIDAS network on the full dataset, which impedes accuracy — and could seriously compromise performance at high levels of missingness.

\section{Technical Details on MIDAS Model and Algorithm}

\section{A Objective Function}

This section offers additional technical details on the MIDAS model's objective function. Recall from the main text that a traditional autoencoder first maps an input vector $\mathrm{x}$ to a lower-dimensional representation $\mathbf{y}$ via a deterministic series of transformations $\mathbf{y}=f_{\theta}(\mathbf{x})$, parameterized by $\theta=\{\mathbf{W}, \mathbf{b}\}$ (Equation 3), and then maps this representation back to a reconstructed vector $\mathbf{z}$ via a converse series of transformations $\mathbf{z}=g_{\theta^{\prime}}(\mathbf{y})$, parameterized by $\theta^{\prime}=\left\{\mathbf{W}^{\prime}, \mathbf{b}^{\prime}\right\}$ (Equation 4 ). Each element $i$ of the input vector $\mathbf{x}_{i}$ is thus mapped to a corresponding element of the hidden representation $\mathbf{y}_{i}$ and the reconstruction $\mathbf{z}_{i}$. The parameters of this model are trained to minimize the average reconstruction error:

$$
\begin{aligned}
\theta^{*}, \theta^{\prime *} & =\underset{\theta^{*}, \theta^{* *}}{\arg \min } \frac{1}{N} \sum_{i=1}^{N} L\left(\mathbf{x}_{i}, \mathbf{z}_{i}\right) \\
& =\underset{\theta^{*}, \theta^{* *}}{\arg \min } \frac{1}{N} \sum_{i=1}^{N} L\left(\mathbf{x}_{i}, g_{\theta^{\prime}}\left(f_{\theta}\left(\mathbf{x}_{i}\right)\right)\right)
\end{aligned}
$$

where $L$ is a loss function (such as a mean squared error function).

In a denoising autoencoder, we again optimize these parameters to minimize the average reconstruction error. Unlike before, however, $\mathbf{z}$ is a deterministic function of $\tilde{\mathbf{x}}$, the 
corrupted input, instead of $\mathbf{x}$. In a MIDAS model, we only seek to minimize the reconstruction error on corrupted values that were originally observed. That is, we want $\mathbf{z}$ to be as close as possible to $\tilde{x}_{\mathrm{obs}}$ (we do not know the original values of $\tilde{x}_{\text {mis }}$ ). If $\mathbf{D}$ consists of two random variables $X$ and $Y$ with joint probability distribution $p(X, Y)$, the overall joint distribution can be characterized as:

$$
q^{0}\left(X, \tilde{X}_{\mathrm{obs}}, \tilde{X}_{\mathrm{mis}}, Y\right)=q^{0}(X) q_{D}\left(\tilde{X}_{\mathrm{obs}}, \tilde{X}_{\mathrm{mis}} \mid X\right) \delta_{f_{\theta}\left(\tilde{X}_{\mathrm{obs}}, \tilde{X}_{\mathrm{mis}}\right)}(Y)
$$

where $q^{0}(X, \tilde{X}, Y)$ is parameterized by $\theta=\{\Omega, \psi\}$. This implies that $Y$ is a deterministic

function of both $\tilde{X}_{\text {obs }}$ and $\tilde{X}_{\text {mis }}$. However, the objective function minimized by stochastic gradient descent only includes the former:

$$
\underset{\theta^{*}, \theta^{\prime *}}{\arg \min } \mathbb{E}_{q^{0}}\left(X, \tilde{X}_{\mathrm{mis}}\right)\left[L\left(X, g_{\theta^{\prime}},\left(f_{\theta}\left(\tilde{X}_{\mathrm{obs}}\right)\right)\right)\right]
$$

The implication of this result is that the MIDAS model minimizes the expected loss over the empirical distribution of not only the observed data but also the subset of corrupted data that were originally observed.

\section{B Training Steps}

As discussed in the main text, a MIDAS network is feedforward: given an initial set of weights and biases, data are propagated forward through the hidden layer of the network and aggregate loss is calculated. Weights and biases are then adjusted via the method of backpropagation. Since the MIDAS network is deep (i.e., it contains more than one hidden layer), this adjustment is made sequentially from the last layer to the first. This section provides a more detailed description of the key training steps in the MIDAS algorithm.

Recall that in the pre-training stage, a missingness indicator matrix $\mathbf{D}$ is generated for 
the input data $\mathbf{D}, \mathbf{D}_{\text {mis }}$ is set to 0 , and a MIDAS network is parameterized using a variant of Xavier Initialization. In each training epoch, we shuffle and divide $\mathbf{D}$ into $B$ mini-batches $\mathcal{B}_{1}, \mathcal{B}_{2}, \ldots, \mathcal{B}_{B}$ of size $s$ (default $s=16$ ); $\mathbf{R}$ is divided into corresponding mini-batches. This step has the advantage of reducing training time - storing all training data in memory and calculating loss for the whole sample are memory-intensive, whereas mini-batches can be processed quickly and in parallel — as well as increasing the frequency of model updates, which ensures more robust convergence (for instance, by avoiding local minima).

In the next step, we partially corrupt the input data by multiplying the $B$ mini-batches by a Bernoulli vector $\mathbf{v}$ with $p=0.8$ (resulting in a corruption rate of $20 \%$ ):

$$
\begin{aligned}
\tilde{\mathbf{x}} & =\left[v^{(0,1)} \mathcal{B}_{1}, \ldots, v^{(0, n)} \mathcal{B}_{B}\right] \\
\mathbf{v}^{(0)} & \sim \operatorname{Bernoulli}(p=0.8)
\end{aligned}
$$

We then implement dropout regularization by partially corrupting nodes in the hidden layers of the network. This involves multiplying outputs from each layer by another Bernoulli vector with $p=0.5$ (a corruption rate of 50\%):

$$
\begin{aligned}
\tilde{\mathbf{y}}^{(h)} & =\mathbf{y}^{(h)} \mathbf{v}^{(h)} \\
\mathbf{v}^{(h)} & \sim \operatorname{Bernoulli}(p=0.5)
\end{aligned}
$$

We then perform a full forward pass through the network - using both the corrupted inputs $\tilde{\mathbf{x}}$ and the corrupted hidden nodes $\tilde{\mathbf{y}}^{(h)}-$ to generate our input reconstruction $\mathrm{z}$ (described in Equations 5 and 6 in the main text). Loss is calculated with respect to the subset of corrupted data that were originally observed ( $\left.\tilde{\mathrm{x}}_{\mathrm{obs}}\right)$, which is achieved by multiplying the RMSE and cross-entropy loss functions by a missingness indicator vector $\mathbf{r}$ (see Equation 7). A weight decay regularization term $\lambda$ is included in the calculation to 
reduce overfitting:

$$
E=L(\mathbf{x}, \mathbf{z}, \mathbf{r})+\lambda\|\mathbb{E}[\mathbf{W}]\|^{2}
$$

In the backpropagation step, we find the gradient of the loss function with respect to the weights of the network.2 Since the change in error with respect to the weights in a given layer $\left(\mathbf{W}^{(h)}\right)$ depends on the weights in the next layer $\left(\mathbf{W}^{(h+1)}\right)$, this must be calculated sequentially from the output layer to the input layer. Specifically, for each layer, we must derive $\frac{\partial E}{\partial \mathbf{W}^{(h)}}$. Through two applications of the chain rule, this problem becomes more tractable:

$$
\begin{aligned}
\frac{\partial E}{\partial \mathbf{W}^{(h)}} & =\frac{\partial E}{\partial \mathbf{y}^{(h)}} \cdot \frac{\partial \mathbf{y}^{(h)}}{\partial \mathbf{W}^{(h)}} \\
& =\frac{\partial E}{\partial \mathbf{y}^{(h+1)}} \cdot \frac{\partial \mathbf{y}^{(h+1)}}{\partial \mathbf{y}^{(h)}} \cdot \frac{\partial \mathbf{y}^{(h)}}{\partial \mathbf{W}^{(h)}},
\end{aligned}
$$

The first term of Equation $\mathrm{A9}$ indicates that the layer-specific partial derivative of the loss function depends on the derivative with respect to outputs from the next layer. The middle term is the partial derivative of the next layer's outputs with respect to the current layer's, which is equivalent to the derivative of the next layer's activation function $\frac{\partial f\left(\mathbf{y}^{(h+1)}\right.}{\mathbf{y}^{(h+1)}}$. Since $\mathbf{y}^{(h)}$ is the weighted sum of the inputs into layer $h$, the right term is simply equal to $\mathbf{y}^{(h-1)}$. Note that the latter two terms are straightforward to derive because the functional form of each layer's activation function is known a priori.

Once errors have been fully backpropagated through the network, we use the calculated gradients to update the MIDAS network's weights. Each weight is adjusted in the direction of the negative gradient, tempered by some learning rate $\gamma$ that stabilizes convergence by scaling the step size according to the application at hand:

$$
\Delta \mathbf{W}^{(h)}=-\gamma \frac{\partial E}{\partial \mathbf{W}^{(h)}}
$$

${ }^{2}$ For a more in-depth discussion of the backpropagation procedure, see Goodfellow et al. (2016, Chapter 6). 
Once all weights are updated, the training epoch is complete. This procedure is repeated iteratively until the loss function converges.

\section{Additional Information on Applied Accuracy Test}

\section{TABLE A1. Summary Statistics for Adult Dataset}

\begin{tabular}{|c|c|c|c|c|}
\hline Variable & Type & Missing & Distribution & Description \\
\hline class_labels (outcome) & Binary & 0 & >50K: 11,$687 ; \leq 50 \mathrm{~K}: 37,155$ & Annual income \\
\hline age & Continuous & 0 & Mean $=38.64 ;$ SD $=13.71$ & Age \\
\hline workclass & $\begin{array}{l}\text { Unordered } \\
\text { categorical }\end{array}$ & 2,799 & $\begin{array}{l}\text { Mode }=\text { Private }(33,906) ; 7 \\
\text { other categories }\end{array}$ & Employment type \\
\hline fnlwgt & Continuous & 0 & $\begin{array}{l}\text { Mean }=189,664 ; \text { SD }= \\
105,604\end{array}$ & $\begin{array}{l}\text { Final weight (expected } \\
\text { number in population) }\end{array}$ \\
\hline education & Ordinal & 0 & $\begin{array}{l}\text { Mode }=\text { HS-grad }(15,784) \\
15 \text { other categories }\end{array}$ & $\begin{array}{l}\text { Highest level of } \\
\text { education (categorical) }\end{array}$ \\
\hline education_num & Continuous & 0 & Mean $=10.08 ;$ SD $=2.57$ & $\begin{array}{l}\text { Highest level of } \\
\text { education (numerical) }\end{array}$ \\
\hline marital_status & $\begin{array}{l}\text { Unordered } \\
\text { categorical }\end{array}$ & 0 & $\begin{array}{l}\text { Mode }=\text { Married-civ-spouse } \\
(22,379) ; 6 \text { other categories }\end{array}$ & Marital status \\
\hline occupation & $\begin{array}{l}\text { Unordered } \\
\text { categorical }\end{array}$ & 2,809 & $\begin{array}{l}\text { Mode }=\text { Prof_speciality } \\
(6,172) ; 13 \text { other categories }\end{array}$ & Employment sector \\
\hline relationship & $\begin{array}{l}\text { Unordered } \\
\text { categorical }\end{array}$ & 0 & $\begin{array}{l}\text { Mode }=\text { Husband }(19,716) ; 5 \\
\text { other categories }\end{array}$ & Position in family \\
\hline race & $\begin{array}{l}\text { Unordered } \\
\text { categorical }\end{array}$ & 0 & Mode $=$ White $(41,762)$ & Race \\
\hline sex & Binary & 0 & $\begin{array}{l}\text { Mode }=\text { Male }(32,650) ; 1 \\
\text { other category }\end{array}$ & Sex \\
\hline capital_gain & Continuous & 0 & $\begin{array}{l}\text { Mean }=1079 ; \text { SD }= \\
7,452.019\end{array}$ & Capital gains \\
\hline capital_loss & Continuous & 0 & Mean $=87.5 ;$ SD $=403.00$ & Capital losses \\
\hline hours_per_week & Continuous & 0 & Mean $=40.42 ; S D=12.39$ & $\begin{array}{l}\text { Hours worked per } \\
\text { week }\end{array}$ \\
\hline native_country & $\begin{array}{l}\text { Unordered } \\
\text { categorical }\end{array}$ & 857 & $\begin{array}{l}\text { Mode }=\text { United-States } \\
(43,832) ; 41 \text { other categories }\end{array}$ & Country of origin \\
\hline
\end{tabular}

The dataset has 48,842 rows representing individuals surveyed in the 1994 United States Census. 
TABLE A2. Missingness Treatments Applied to Adult Dataset

\begin{tabular}{|c|c|c|}
\hline $\begin{array}{l}\text { Missingness } \\
\text { Pattern }\end{array}$ & Step & Procedure to Obtain $R$ (Missingness Indicator Vector) \\
\hline MCAR & 1. & $\begin{array}{l}\text { Randomly select proportion of columns }(0.3,0.5,0.7 \text {, or } 0.9) \text { for miss- } \\
\text { ingness treatment. native_country, occupation, and education cannot } \\
\text { be selected (due to computational issues with Amelia). } \\
R \sim \operatorname{Bernoulli}(p=0.5) \text { for each selected column. }\end{array}$ \\
\hline \multirow[t]{3}{*}{ MAR } & $\begin{array}{l}1 . \\
2 .\end{array}$ & $\begin{array}{l}\text { MCAR step } 1 . \\
L=\text { one column randomly sampled from those not selected (latent }\end{array}$ \\
\hline & 3a. & $\begin{array}{l}\text { If } L \text { is continuous, select all rows with values at or below median of } L \text {. } \\
\text { Sample N/2 rows from this matrix. For each selected column, } R_{i}=1 \\
\text { if row } i \text { 's value is in this sample. }\end{array}$ \\
\hline & $3 \mathrm{~b}$. & $\begin{array}{l}\text { If } L \text { is categorical, randomly sample half of all categories. If no. of } \\
\text { rows in this matrix }>50 \% \text { of } \mathrm{N} \text {, sample } \mathrm{N} / 2 \text { of rows. For each selected } \\
\text { column, } R_{i}=1 \text { for all rows in remaining sample. }\end{array}$ \\
\hline \multirow[t]{4}{*}{ MNAR } & 1. & MCAR step 1. \\
\hline & 2. & $L=$ selected column \\
\hline & 3a. & If $L$ is continuous, MAR step $3 \mathrm{a}$. \\
\hline & $3 b$. & $\begin{array}{l}\text { If } L \text { is categorical, select modal category. For each selected column, } \\
R_{i}=1 \text { for all except randomly sampled } 5 \% \text { percent of this sample. }\end{array}$ \\
\hline
\end{tabular}




\section{Additional Information on Scalability Analysis}

\section{A List of Variables in Column-Wise Test}

Binary gender, pew_bornagain, cit1, investor, trans, votereg, edloan, CC18_417a_1, CC18_417a_2, CC18_417a_3, CC18_417a_4,CC18_417a_5, CC18_417a_6, CC18_417a_7, CC18_417a_8, CC18_418a,CC18_414A, CC18_414B, CC18_414C, CC18_414D, CC18_414E, CC18_324a, CC18_324b,CC18_324c,CC18_324d, CC18_415a, CC18_415b, CC18_415c, CC18_415d, CC18_416, CC18_417_a, CC18_417_b, CC18_417_c, CC18_417_d, CC18_417_e, healthins_1, healthins_2, healthins_3, healthins_4, healthins_5, healthins_6, healthins_7, CC18_300_1, CC18_300_2,CC18_300_3, CC18_300_4, CC18_300_5,CC18_300_6, CC18_303_1,CC18_303_2, CC18_303_3,CC18_303_4,CC18_303_5, CC18_303_6, CC18_303_7,CC18_303_8,CC18_303_9, CC18_303_10,CC18_303_11,CC18_320a,CC18_320c,CC18_320d,CC18_321a,CC18_321b, CC18_321c,CC18_321d,CC18_322a, CC18_322b, CC18_322c_new, CC18_322d_new, CC18_322c, CC18_322f, CC18_325a,CC18_325b, CC18_325c,CC18_325d, CC18_325e_new, CC18_325f_new, CC18_326, CC18_327a, CC18_327c, CC18_327d, CC18_327e, CC18_328b, CC18_328d, CC18_328e,CC18_328f, CC18_331a,CC18_331b,CC18_331c,CC18_332a, CC18_332b, CC18_332c, CC18_332e

Categorical sexuality, educ, race, employ, internethome, internetwork, marstat, pid3, religpew, ownhome, urbancity, immstat, union_coverage, unionhh, CC18_309a, CC18_309b, CC18_309c, CC18_309d, CC18_316, CC18_318a, CC18_335, CC18_350

Ordinal pew_religimp, pid7, ideo5, pew_churatd, pew_prayer, newsint, faminc_new, CC18_421a, CC18_app_dtrmp_post, CC18_422a, CC18_422b, CC18_422c, CC18_422d, CC18_422e, CC18_422f, CC18_422g, CC18_426_1, CC18_426_2, CC18_426_3, CC18_426_4, CC18_426_5, CC18_427_a, CC18_427_b, CC18_427_c, CC18_427_d, CC18_302 
Continuous birthyr, citylength_1

\section{B List of Variables in Row-Wise Test}

Binary gender, pew_bornagain, cit1, investor, trans, votereg

Categorical sexuality, educ, internethome, internetwork, marstat, pid3, ownhome, urbancity, immstat, unionhh

Ordinal pew_religimp, pid7, ideo5, pew_churatd, pew_prayer, newsint, faminc_new

Continuous birthyr, citylength_1

\section{Additional Information on Latent Ideology Estimation}

\section{A List of Policy Questions}

As discussed in the main text, we estimate CCES respondents' latent ideology by regressing their ideological self-placement on their answers to 19 policy questions in the survey. The former is based on CCES question CC18_334A (Ideological Placement - Yourself): "How would you rate each of the following individuals and groups?" Response options range from 1 for "Very Liberal" to 7 for "Very Conservative." The 19 policy variables are listed in Table A3,

\section{B Comparison with Amelia}

Although existing MI algorithms cannot accommodate the full CCES sample on which we train the MIDAS imputation model, some of them can handle small subsets of this sample 
TABLE A3. List of CCES Policy Variables Included in Latent Ideology Estimation

\begin{tabular}{llll}
\hline Variable & Policy Area & Response Type & Missing \\
\hline CC18_414A & Minimum Wage & For/Against & 8202 \\
CC18_414B & Millionaire's tax & For/Against & 8216 \\
CC18_414C & Sales tax & For/Against & 8233 \\
CC18_414D & Income tax & For/Against & 8230 \\
CC18_414E & Abortion spending & For/Against & 8223 \\
CC18_324a & Government Spending & Support/Oppose & 8304 \\
CC18_324b & Government Spending & Support/Oppose & 8324 \\
CC18_324c & Government Spending & Support/Oppose & 8298 \\
CC18_324d & Government Spending & Support/Oppose & 8280 \\
CC18_415a & Carbon Dioxide regulation & Support/Oppose & 8517 \\
CC18_415b & Fuel efficiency regulation & Support/Oppose & 8499 \\
CC18_415c & Renewable energy policy & Support/Oppose & 8476 \\
CC18_415d & EPA powers & Support/Oppose & 8456 \\
CC18_416 & Financial regulation & Support/Oppose & 8495 \\
CC18_426_1 & State welfare spending & Increase/Decrease (1-5) & 8311 \\
CC18_426_2 & State healthcare spending & Increase/Decrease (1-5) & 8330 \\
CC18_426_3 & State education spending & Increase/Decrease (1-5) & 8353 \\
CC18_426_4 & State law enforcement spending & Increase/Decrease (1-5) & 8380 \\
CC18_426_5 & State transportation/infrastructure & Increase/Decrease (1-5) & 8364 \\
& spending & & \\
\hline
\end{tabular}

that exclude categorical variables with a large number of levels. Importantly, however, some of these omitted variables - such as respondents' state of residence and religion — are likely to be strong predictors of both the policy items and missingness in these variables. When they are excluded from the imputation model, therefore, estimates of latent ideology will tend to be closer to those based on listwise deletion.

To illustrate this point, we estimate latent ideology using a subset of the CCES data with the Amelia package in R. Specifically, we include five demographic variables - gender, sexuality, race, sector of employment, and party identification - in addition to the 19 policy variables included in the regression model (Equation 8) 3 As with MIDAS, we then generate 15 completed datasets and recover latent ideology estimates from the fitted

\footnotetext{
${ }^{3}$ We exclude several demographic variables with a higher number of categories to enable convergence.
} 
FIGURE A1. Comparison of Latent Ideology Estimates from Different MI Strategies

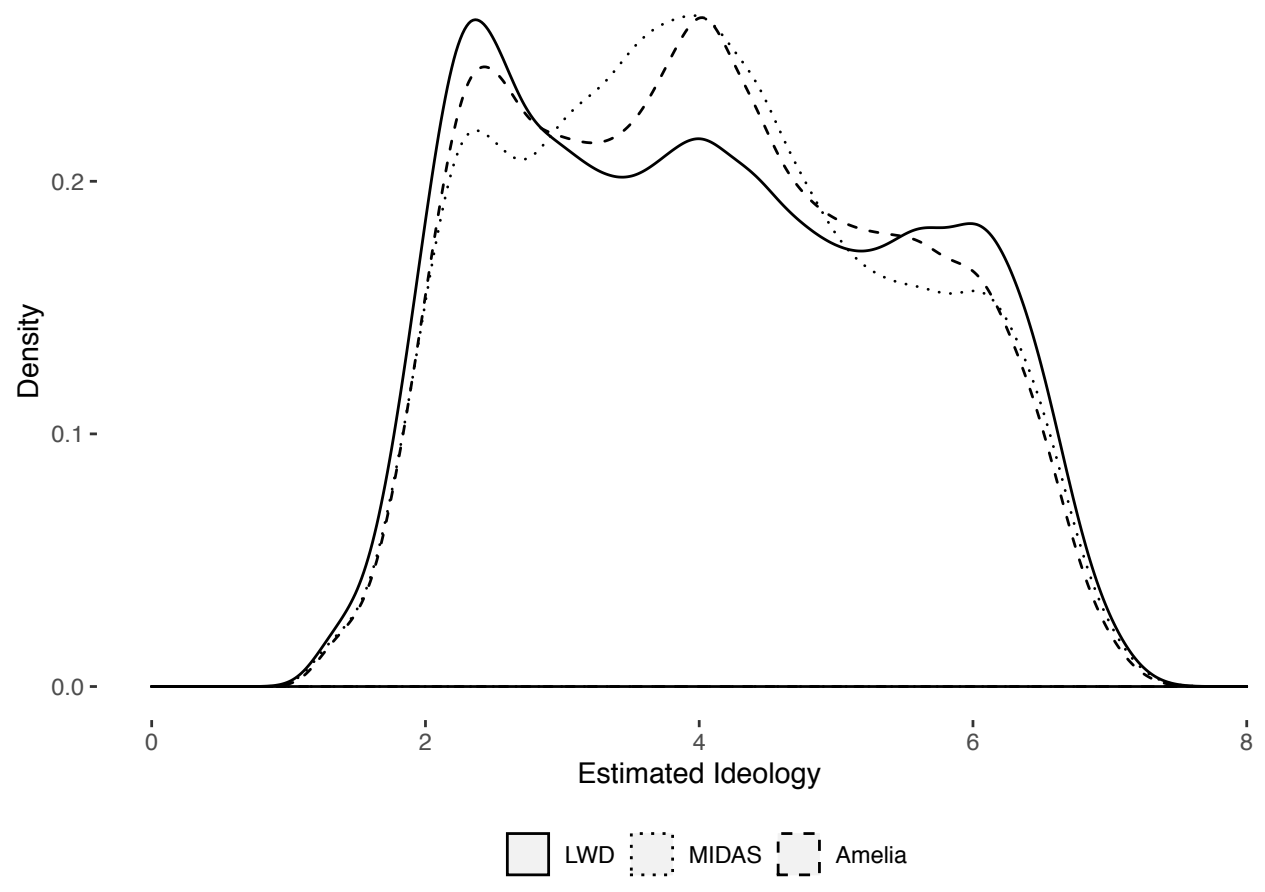

values of the regression.

Figure A1 plots the latent ideology estimates from listwise deletion, MIDAS, and Amelia. As expected, Amelia's estimates are substantially closer to the listwise deletion estimates than MIDAS's. While the modal category is 4, there is a more pronounced peak on the left (liberal) side of the ideology scale and a flatter tail on the right (conservative) side. Compared to MIDAS, therefore, Amelia yields estimates with a clearly more peaked and less normal shape. We can reject the null hypothesis that the three sets of estimates are drawn from the same distribution at the $p<0.01$ level in Kolmogorov-Smirnov tests.

These inferential differences are also significant from a practical perspective. In real datasets such as the CCES, the pattern and specific determinants of missingness are not known. The best option for users of MI is to leverage as much predictive information about the missingness mechanism and incomplete variables as possible. MIDAS enables us to utilize considerably more such information than existing MI strategies - with no 
loss in imputation speed or accuracy — reducing the risk of bias and increasing statistical efficiency.

\section{Imputing Time-Series Cross-Sectional Data: An Illustration}

Finally, this appendix provides an illustration of MIDAS's ability to handle a particularly common type of non-exchangable data in social science research: time-series cross-sectional data.4 As Honaker and King (2010) note, the dominant approach to MI tends to perform poorly with such data, yielding imputed values that are implausible based on substantive knowledge or that deviate substantially from previous and subsequent observations in a smoothly varying time series. These problems arise because the approach "assumes that the missing values are linear functions of other variables' observed values, observations are independent conditional on the remaining observed values, and all the observations are exchangable in that the data are not organized in hierarchical structures" (Honaker and King 2010, 565).5 Although MIDAS — like most MI strategies — does not include any special functionalities for non-exchangable data, we have found that its capacity to learn complex relationships among variables enables it to accurately impute values in time-series cross-sectional settings with only small adjustments to the imputation model.

Building on an experiment conducted by Honaker and King (2010, 565-569) with Amelia, we demonstrate this capacity using data from the World Bank's World Development Indicators (WDI), a collection of almost 1,600 time-series indicators of social and economic development covering 217 countries since 1960.6 We select six African countries - Cameroon, Côte D'Ivoire, Congo Republic, Ghana, Niger, and Zambia - over the

\footnotetext{
${ }^{4}$ Data are non-exchangeable if observations cannot be reordered without altering their joint distribution. More formally, a sequence of random variables $X_{1}, X_{2}, \ldots, X_{n}$ is non-exchangeable if its joint distribution is not identical to that of any (finite) permutation of its indices: $p\left(X_{1}, X_{2}, \ldots, X_{n}\right) \neq p\left(X_{\pi(1)}, X_{\pi(2)}, \ldots, X_{\pi(n)}\right)$. ${ }^{5}$ Amelia seeks to avoid these problems by allowing users to construct a general model of temporal patterns with a sequence of polynomials of the time index. Such a sequence could, of course, be included in a MIDAS model.

6 http://datatopics.worldbank.org/world-development-indicators/.
} 
period 1970-2000, drop all entirely missing columns, and sequentially remove a single country-year observation of GDP (measured in constant 2010 United States dollars) from each cross-section (31 years $\times$ six countries). This yields 186 different subsets of the WDI, each comprising 186 observations and 1251 variables — samples that are too wide for any existing MI algorithm to process. Note, however, does this setup does not play to MIDAS's strengths either, given that the accuracy of neural networks generally increases with the number of observations.

For each sample, we generate lags and leads of all (non-index) variables, since both past and future values of a given variable tend to be correlated with its present value (Honaker et al. 2011, 19). Based on an overimputation analysis (see Section 21), we instantiate MIDAS with two hidden layers of 1024 and 512 nodes, a learning rate of $3 e-5$, a dropout rate of 0.95 , and 2000 training epochs. We include country dummies as well as the lags and leads in the imputation models, bringing the total number of variables to 3756 200 completed datasets are then produced with each model.

Figure A2 compares real versus MIDAS-imputed values of GDP for the six countries. In general, the latter data track the former remarkably closely through each time series, even capturing trends that were missed by Amelia, such as Côte d'Ivoire's cocoa crisis in the late 1970s and Cameroon's strong economic recovery in the mid-1980s (Honaker and King 2010, 569). Only a handful of real values fall outside the interquartile range of MIDAS's imputations, most of which are at the extremities of the time series. This is probably a consequence of the absence both of lags at the beginning of the time series and of leads at the end. Incorporating into the imputation model data from shortly before and after the time period of interest — if available — may help to avoid this problem.

\footnotetext{
${ }^{7}$ We deviate from Honaker and King s selection of countries by substituting Niger for Mozambique, since the latter lacks a complete GDP time series in the WDI.

${ }^{8}$ Given the large number of imputation models in this exercise, we pass all variables other than country, year, and GDP to the additional_data argument in MIDAS, which excludes them from the cost function and hence accelerates training.
} 
FIGURE A2. Real Versus MIDAS-Imputed GDP for Six African Countries, 1970-2000
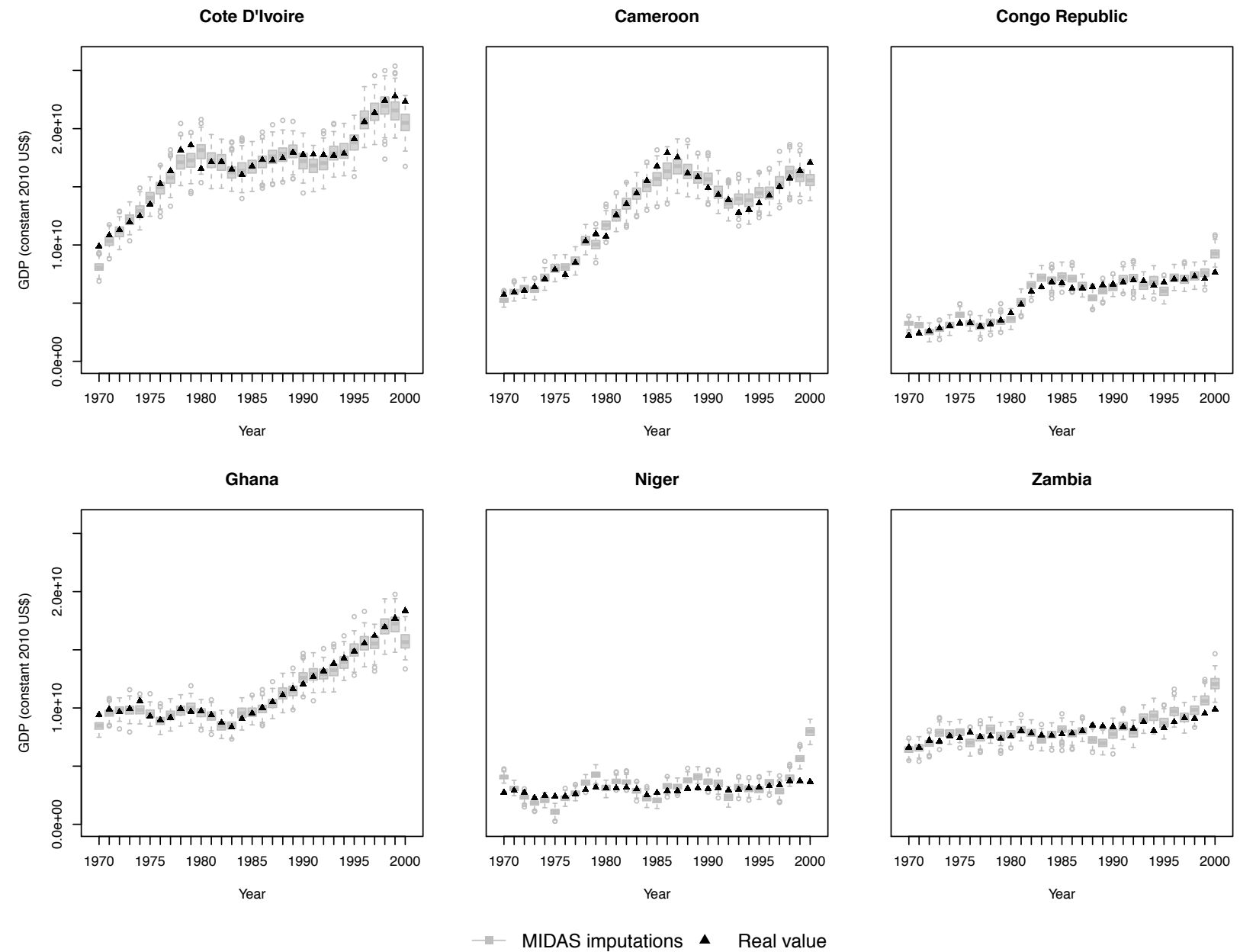

MIDAS imputations are based on variants of the WDI dataset in which country-year observations of GDP are sequentially removed. Each imputation model includes all variables in the WDI that are not entirely missing for the six countries, leads and lags of all non-index variables, and country dummies.

In sum, MIDAS can successfully recover smooth temporal trends in GDP for all six countries. This is particularly notable in light of the absence of explicit features for modeling time and the high ratio of variables to observations, which often leads to poor imputation accuracy with existing MI strategies. To be sure, MIDAS would not perform as well in the presence of longer periods of missingness and sharper inflection points in the time series. However, provided that the imputation model contains sufficiently rich information about 
how observed values are related at different points in time, posterior uncertainty should be low enough to permit valid statistical inference. The inclusion of additional features in the model, such as polynomials of the time index and flexible basis functions, could further improve MIDAS's performance.

\section{References}

Blackwell, Matthew , James Honaker, and Gary King (2017). A unified approach to measurement error and missing data: Overview and applications. Sociological Methods \& Research 46 (3), 303-341.

Christiansen, Bo (2005). The shortcomings of nonlinear principal component analysis in identifying circulation regimes. Journal of Climate 18(22), 4814-4823.

Goodfellow, Ian, Yoshua Bengio, and Aaron Courville (2016). Deep Learning. Cambridge, MA: The MIT Press.

Honaker, James and Gary King (2010). What to do about missing values in time-series cross-section data. American Journal of Political Science 54 (2), 561-581.

Honaker, James , Gary King, and Matthew Blackwell (2011). Amelia II: A program for missing data. Journal of Statistical Software 45 (7), 1-47.

Kingma, Diederik P. and Max Welling (2013). Auto-encoding variational bayes. ArXiv e-prints.

Probst, Philipp , Anne-Laure Boulesteix, and Bernd Bischl (2019). Tunability: Importance of hyperparameters of machine learning algorithms. Journal of Machine Learning Research 20(53), 1-32.

Rezende, Danilo Jimenez, Shakir Mohamed, and Daan Wierstra (2014). Stochastic backpropagation and approximate inference in deep generative models. In International Conference on Machine Learning, pp. 1278-1286. ACM.

Scholz, Matthias (2012). Validation of nonlinear pca. Neural Processing Letters 36 (1), 21-30. 